\title{
Alpha-alpha scattering in the Multiverse
}

\author{
Serdar Elhatisari, ${ }^{a}$ Timo A. Lähde, ${ }^{b}$ Dean Lee, ${ }^{c}$ Ulf-G. Meißner ${ }^{d, e, f}$ and Thomas Vonk ${ }^{d}$ \\ ${ }^{a}$ Faculty of Natural Sciences and Engineering, Gaziantep Islam Science and Technology University, \\ Gaziantep 27010, Turkey \\ ${ }^{b}$ Institute for Advanced Simulation, Institut für Kernphysik, \\ Center for Advanced Simulation and Analytics, and Jülich Center for Hadron Physics, \\ Forschungszentrum Jülich, D-52425 Jülich, Germany \\ ${ }^{c}$ Facility for Rare Isotope Beams and Department of Physics and Astronomy, \\ Michigan State University, East Lansing, MI 48824, U.S.A. \\ ${ }^{d}$ Helmholtz-Institut für Strahlen- und Kernphysik and Bethe Center for Theoretical Physics, \\ Universität Bonn, D-53115 Bonn, Germany \\ ${ }^{e}$ Institute for Advanced Simulation, Institut für Kernphysik, \\ Center for Advanced Simulation and Analytics, and Jülich Center for Hadron Physics, \\ Forschungszentrum Jülich, D-52425 Jülich, Germany \\ ${ }^{f}$ Tbilisi State University, 0186 Tbilisi, Georgia \\ E-mail: selhatisari@gmail.com, t.laehde@fz-juelich.de, \\ leed@frib.msu.edu, meissner@hiskp.uni-bonn.de, vonk@hiskp.uni-bonn.de
}

ABSTRACT: We investigate the phase shifts of low-energy $\alpha$ - $\alpha$ scattering under variations of the fundamental parameters of the Standard Model, namely the light quark mass, the electromagnetic fine-structure constant as well as the QCD $\theta$-angle. As a first step, we recalculate $\alpha$ - $\alpha$ scattering in our Universe utilizing various improvements in the adiabatic projection method, which leads to an improved, parameter-free prediction of the S- and D-wave phase shifts for laboratory energies below $10 \mathrm{MeV}$. We find that positive shifts in the pion mass have a small effect on the S-wave phase shift, whereas lowering the pion mass adds some repulsion in the two-alpha system. The effect on the D-wave phase shift turns out to be more pronounced as signaled by the D-wave resonance parameters. Variations of the fine-structure constant have almost no effect on the low-energy $\alpha$ - $\alpha$ phase shifts. We further show that up-to-and-including next-to-leading order in the chiral expansion, variations of these phase shifts with respect to the QCD $\theta$-angle can be expressed in terms of the $\theta$-dependent pion mass.

Keywords: Chiral Lagrangian, Other Lattice Field Theories, Flavour Symmetries

ArXiv EPrint: 2112.09409 


\section{Contents}

1 Introduction 1

2 Basic concepts 3

3 Pion mass dependence of the nuclear Hamiltonian 5

4 Dependence of the nuclear Hamiltonian on the fine-structure constant 8

5 Theta-dependence of alpha-alpha scattering 9

6 Adiabatic projection method and auxiliary field quantum Monte Carlo simulations

7 Extracting scattering phase shifts from the adiabatic matrices

8 Results 14

$\begin{array}{ll}8.1 \text { Our universe } & 14\end{array}$

$\begin{array}{ll}8.2 \text { The Multiverse } & 15\end{array}$

$\begin{array}{lll}\text { 8.2.1 Variations of the bound state energies } & 15\end{array}$

$\begin{array}{ll}\text { 8.2.2 Pion mass variations of alpha-alpha scattering } & 17\end{array}$

8.2.3 Alpha-alpha scattering with varying $\alpha_{\mathrm{EM}} \quad 20$

8.2.4 Remarks on the $\theta$-dependence of alpha-alpha scattering 21

$9 \quad$ Summary and outlook $\quad 22$

A Details on the NN scattering parameters at varying pion mass 23

B Euclidean time extrapolation $\quad 24$

C The Coulomb modified ERE

D Bound state energies for varying pion masses $\quad 25$

\section{Introduction}

Alpha-alpha $(\alpha-\alpha)$ scattering is one of the most fundamental reactions in nuclear (astro)physics. It is the basic component of the triple-alpha $(3 \alpha)$ reaction prevalent in hot old stars, that leads to the generation of ${ }^{12} \mathrm{C}$ and successively ${ }^{16} \mathrm{O}$, where the ${ }^{12} \mathrm{C}$ production is enhanced through a $J^{P}=0^{+}$resonance at $7.65 \mathrm{MeV}$ excitation energy close to the $3 \alpha$-threshold, the famous Hoyle state [1]. $\alpha$ - $\alpha$ scattering itself features some fine-tuning, as the large 
near-threshold S-wave results from a state with $\left(J^{P}, I\right)=\left(0^{+}, 0\right)$ at an energy $E_{R} \simeq 0.1 \mathrm{MeV}$ above the threshold, see e.g. the review [2], with a tiny width of $\Gamma_{R} \simeq 6 \mathrm{eV}$. It is precisely this small width (long lifetime) of the unstable ${ }^{8} \mathrm{Be}$ nucleus that allows for the reaction with the third $\alpha$ particle in the $3 \alpha$ reaction at sufficiently high temperatures and densities.

The fine-tunings in these (and other) fundamental nuclear reactions together with other fine-tunings in particle physics and cosmology have led to the concept of the Multiverse, where our Universe with its observed values is part of a larger structure of universes featuring different sets of the fundamental constants. Related to this are anthropic considerations, which is the philosophical idea that the parameters governing our world should fit the intervals compatible with the existence of life on Earth. More details can be found in the reviews [3-7].

Coming back to nuclear physics, the closeness of the Hoyle state energy to the $3 \alpha$ threshold invites investigations about the stability of this resonance condition under changes of the fundamental parameters of the strong and the electromagnetic (EM) interactions, whose interplay guarantees the stability of atomic nuclei. While earlier investigations, see e.g. ref. [8], suffered from some model-dependence in the description of the nuclear forces, using the $a b$ initio method of Nuclear Lattice Effective Field Theory (NLEFT) this topic was re-investigated in refs. [9-11]. More specifically, the quark mass dependence as well as the dependence on the electromagnetic fine-structure constant of the nuclear Hamiltonian was worked out, using and combining results from chiral perturbation theory (CHPT) and lattice QCD simulations for the pion decay constant, the nucleon mass and so on. Here, we will use the same chiral EFT at next-to-next-to-leading order combined with the socalled Adiabatic Projection Method (APM), that allows for ab initio calculations of nuclear reactions, as developed in refs. [12-14]. Using the APM, the scattering of two alpha clusters has been achieved on the lattice [15], enabled by the fact that the computational effort is approximately quadratic in the number of nucleons in the scattering clusters. The method was further refined in ref. [16]. Combining these different works, we are thus in the position to investigate the sensitivity of the low-energy $\alpha$ - $\alpha$ phase shifts on variations in the light quark mass $\hat{m}$ and the EM fine-structure constant $\alpha_{\mathrm{EM}}$. We note that $\alpha$ - $\alpha$ scattering has also recently been studied using the no-core shell model within a continuum approach [17].

While the investigation of the resonance enhancement in the $3 \alpha$ process due to the Hoyle state already sets rather stringent limits on the possible variations of the light quark mass and the fine-structure constant, one has to be aware that these results are afflicted with some inherent uncertainties, as in the corresponding stellar simulations only the distance of the Hoyle state to the $3 \alpha$-threshold is varied. Translating this into a dependence on, say, the light quark mass assumes that only the nuclei directly involved in the $3 \alpha$ process are subject to these changes, but of course one should perform the complete stellar simulations (reaction networks) with appropriately modified masses and reaction rates. At present, this is only possible for Big Bang Nucleosynthesis, see e.g. refs. [18, 19], but not for the whole nuclear reaction networks in stars. Therefore, the ab initio computation of the dependence of $\alpha$ - $\alpha$ scattering on the fundamental parameters of the Standard Model is not subject to such uncertainties and paves the way for more elaborate network calculations in the Multiverse.

A parameter that has obtained less attention in such anthropic considerations is the QCD $\theta$-term, as the bounds from the neutron electric dipole moment require $\theta \lesssim 10^{-10}$, 
see e.g. ref. [20] for a recent lattice QCD study. Still, it is worth to reconsider bounds on the $\theta$-angle from observations other than the neutron EDM as well as from anthropic considerations, as done e.g. in refs. [21, 22]. In particular, it was shown in [22] that nuclear binding increases with $\theta$ and that $\theta \lesssim 0.1$ would not upset the world as we know it. It is thus also of interest to study the reaction rate of the fundamental $\alpha$ - $\alpha$ scattering process as a function of $\theta$, as will be done here.

In ref. [23], it was shown that symmetric nuclear matter without Coulomb interactions lies close to a quantum phase transition between a Bose gas of alpha clusters and a nuclear liquid. Whether one is in the Bose gas phase or the nuclear liquid phase is determined by the sign of the $\alpha-\alpha$ S-wave scattering length. In turn, the $\alpha$ - $\alpha$ scattering phase shifts depend on the strength, range, and locality of the nucleon-nucleon interactions. The nucleon-nucleon interactions need enough attractive strength, range, and locality to overcome the Pauli repulsion between nucleons with the same spin and isospin [24, 25]. Locality here refers to interactions that are diagonal when written in position space. The variation of the light quark masses, electromagnetic fine-structure constant, and $\theta$ parameter will produce changes to the leading-order interactions, and we take these changes to the nucleon-nucleon interactions to be local. This choice is motivated by studies of Quantum Chromodynamics in the limit of a large number of colors showing that the nucleon-nucleon interactions reduce to local interactions with an underlying spin-isospin exchange symmetry [26-28].

The paper is organized as follows. In section 2, we introduce the dependence of the two-alpha cluster energy on the fundamental parameters of the Standard Model, the basic framework of NLEFT and give a first glimpse on some of the relevant quark (pion) mass dependences. The pion mass dependence of the nuclear Hamiltonian used here is presented in detail in section 3. Then, in section 4 we discuss the inclusion of the electromagnetic interaction and the dependence of the nuclear Hamiltonian on the fine-structure constant. Section 5 shows how the $\theta$-dependence of $\alpha$ - $\alpha$ scattering can be inferred from the $\theta$ dependence of the pion mass. In section 6 we collect the computational tools needed for this investigations. We give the basic APM formalism needed for our investigation and show how various quantities are obtained from Auxiliary Field Quantum Monte Carlo simulations. In section 7 , we show how to extract the scattering phase shifts from the adiabatic transfer matrices. Our results are presented and discussed in section 8. We end with a summary and conclusions. Some further details of the computations are relegated to the appendices.

\section{Basic concepts}

We aim to compute the variation of the $\alpha-\alpha$ scattering phase shifts as a function of the fundamental constants of nature following refs. $[10,11]$. Since we compute the scattering phase shifts from the spectrum, we consider a linear variation in the light quark mass and the electromagnetic fine-structure constant $\alpha_{\mathrm{EM}}$ of the two-alpha cluster energy,

$$
\left.\delta E_{\alpha \alpha} \simeq \frac{\partial E_{\alpha \alpha}}{\partial M_{\pi}}\right|_{M_{\pi}^{\mathrm{ph}}} \delta M_{\pi}+\left.\frac{\partial E_{\alpha \alpha}}{\partial \alpha_{\mathrm{EM}}}\right|_{\alpha_{\mathrm{EM}}^{\mathrm{ph}}} \delta \alpha_{\mathrm{EM}},
$$


where we have used the Gell-Mann-Oakes-Renner relation, $M_{\pi}^{2}=2 B_{0} \hat{m}$, with $\hat{m}=\left(m_{u}+\right.$ $\left.m_{d}\right) / 2$ the light quark mass and $B_{0}$ is related to the scalar quark condensate. ${ }^{1}$ Throughout, we work in the isospin limit as strong isospin breaking effects are expected to be very small. Further, the superscript "ph" denotes the pertinent values in Nature (the physical world). We note that this formula is applicable for changes in the modulus of the pion mass $\left|\delta M_{\pi} / M_{\pi}\right|$ and the electromagnetic fine-structure constant by $\left|\delta \alpha_{\mathrm{EM}} / \alpha_{\mathrm{EM}}\right| \lesssim 10 \%$. The variation with respect to the QCD $\theta$ angle will be discussed later in a separate section.

Our computational framework is NLEFT, see refs. $[29,30]$ for details. In what follows, we employ a periodic cubic lattice with a spatial lattice spacing of $a=1.97 \mathrm{fm}$ and a temporal lattice spacing $a_{t}=1.32 \mathrm{fm}$. For free nucleons we use the $\mathcal{O}\left(a^{4}\right)$-improved lattice Hamiltonian,

$$
\begin{aligned}
H_{\text {free }}= & \frac{49}{12 m_{N}} \sum_{\vec{n}} \sum_{i, j=0,1} a_{i, j}^{\dagger}(\vec{n}) a_{i, j}(\vec{n}) \\
& -\frac{3}{4 m_{N}} \sum_{\vec{n}} \sum_{i, j=0,1} \sum_{l=1,2,3}\left[a_{i, j}^{\dagger}(\vec{n}) a_{i, j}(\vec{n}+\hat{l})+a_{i, j}^{\dagger}(\vec{n}) a_{i, j}(\vec{n} \hat{l})\right] \\
& -\frac{3}{40 m_{N}} \sum_{\vec{n}} \sum_{i, j=0,1} \sum_{l=1,2,3}\left[a_{i, j}^{\dagger}(\vec{n}) a_{i, j}(\vec{n}+2 \hat{l})+a_{i, j}^{\dagger}(\vec{n}) a_{i, j}(\vec{n}-2 \hat{l})\right] \\
& -\frac{1}{180 m_{N}} \sum_{\vec{n}} \sum_{i, j=0,1} \sum_{l=1,2,3}\left[a_{i, j}^{\dagger}(\vec{n}) a_{i, j}(\vec{n}+3 \hat{l})+a_{i, j}^{\dagger}(\vec{n}) a_{i, j}(\vec{n}-3 \hat{l})\right],
\end{aligned}
$$

where $\vec{n}$ represents the integer-valued lattice sites, $m_{N}$ is the nucleon mass, $\hat{l}=\hat{1}, \hat{2}, \hat{3}$ are unit lattice vectors in the spatial directions, $i(j)$ is a spin (isospin) index, and $a_{i, j}$ and $a_{i, j}^{\dagger}$ denote nucleon annihilation and creation operators.

For the leading-order ( $\mathrm{LO}$ ) nuclear interaction we use an improved action which is based on the following nucleon-nucleon (NN) scattering amplitude,

$$
\begin{aligned}
\mathcal{A}_{\mathrm{LO}}= & C_{S=0, I=1} f(\vec{q})\left(\frac{1}{4}-\frac{1}{4} \vec{\sigma}_{i} \cdot \vec{\sigma}_{j}\right)\left(\frac{3}{4}+\frac{1}{4} \vec{\tau}_{i} \cdot \vec{\tau}_{j}\right) \\
& +C_{S=1, I=0} f(\vec{q})\left(\frac{3}{4}+\frac{1}{4} \vec{\sigma}_{i} \cdot \vec{\sigma}_{j}\right)\left(\frac{1}{4}-\frac{1}{4} \vec{\tau}_{i} \cdot \vec{\tau}_{j}\right) \\
& +\tilde{g}_{\pi N}^{2} \vec{\tau}_{i} \cdot \vec{\tau}_{j} \frac{\left(\vec{\sigma}_{i} \cdot \vec{q}\right)\left(\vec{\sigma}_{j} \cdot \vec{q}\right)}{\vec{q}^{2}+M_{\pi}^{2}}
\end{aligned}
$$

where $\vec{\sigma}$ and $\vec{\tau}$ denote the Pauli spin and isospin matrices, $\tilde{g}_{\pi N}$ is the strength of the one-pion-exchange (OPE) potential defined as $\tilde{g}_{\pi N}=g_{A} /\left(2 F_{\pi}\right)$ in terms of the nucleon axial-vector coupling $g_{A}=1.273(19)$ and the pion decay constant $F_{\pi}=92.1 \mathrm{MeV} . C_{S=0, I=1}$ and $C_{S=1, I=0}$ are the coupling constants of the short-range part of the nuclear force which are adjusted to reproduce the scattering phase shifts for the two S-wave channels, and $f(\vec{q})$ is a smearing function which is defined to reproduce the effective ranges for the two S-wave channels. We redefine the low-energy constants (LECs) of the short-range interactions in

\footnotetext{
${ }^{1}$ Because of this relation, we can equivalently use the wordings "quark mass dependence" and "pion mass dependence".
} 
terms of linear combinations of $C_{0}$ and $C_{I}$,

$$
\begin{aligned}
& C_{0}=\frac{3}{4} C_{S=0, I=1}+\frac{1}{4} C_{S=1, I=0}, \\
& C_{I}=\frac{1}{4} C_{S=0, I=1}-\frac{3}{4} C_{S=1, I=0} .
\end{aligned}
$$

From eqs. (2.2) and (2.3) it is obvious that the sources of implicit $M_{\pi}$-dependence are the nucleon mass $m_{N}$, the coupling constant of the OPE potential $\tilde{g}_{\pi N}$, and the LECs of the short-range interactions $C_{0}$ and $C_{I}$, besides the explicit pion mass dependence in the OPE. Before discussing these in detail in section 3, let us consider the quark (pion) mass dependence of the nucleon mass and the pion decay constant to get an idea about the changes we can expect. At the leading one-loop order $\mathcal{O}\left(p^{3}\right)$, where $p$ is a generic small parameter, the chiral expansion of the nucleon mass can be written as

$$
m_{N}\left(M_{\pi}\right)=m_{0}-4 c_{1} M_{\pi}^{2}-\frac{3 g_{A}^{2}\left(M_{\pi}\right) M_{\pi}^{3}}{32 \pi F_{\pi}^{2}\left(M_{\pi}\right)}+\mathcal{O}\left(M_{\pi}^{4}\right),
$$

where $m_{0} \simeq 865 \mathrm{MeV}$ [31] is the nucleon mass in the (two-flavor) chiral limit and $c_{1}=$ $-1.1 \mathrm{GeV}^{-1}$ is a LEC from the chiral pion-nucleon Lagrangian at next-to-leading order (NLO) [32]. Note that the leading correction of order $M_{\pi}^{2}$ is intimately linked to the pion-nucleon $\sigma$-term discussed below. At third order, the pion mass dependence of the pion decay constant and the axial-vector coupling constant is made explicit. For the pion decay constant we use the expression from the chiral expansion at NLO,

$$
F_{\pi}\left(M_{\pi}\right)=F+\frac{M_{\pi}^{2}}{16 \pi^{2} F} \bar{l}_{4}+\mathcal{O}\left(M_{\pi}^{4}\right)
$$

where $F=86.2 \mathrm{MeV}$ is the pion decay constant in the (two-flavor) chiral limit, ${ }^{2}$ and $\bar{l}_{4}=4.3$ is a LEC, where we use the value from ref. [33] (which is consistent with more modern determinations). We postpone the discussion of the nucleon axial-vector coupling $g_{A}$ and of the LECs $C_{0}, C_{I}$ to the next section.

\section{Pion mass dependence of the nuclear Hamiltonian}

First, let us collect the knowledge about the pion mass dependence of the nuclear Hamiltonian. Specifically, the dependence of the energy $E_{\alpha \alpha}$ on the pion mass $M_{\pi}$ can be expressed as

$$
E_{\alpha \alpha}=E_{\alpha \alpha}\left(\tilde{M}_{\pi}, m_{N}\left(M_{\pi}\right), \tilde{g}_{\pi N}\left(M_{\pi}\right), C_{0}\left(M_{\pi}\right), C_{I}\left(M_{\pi}\right)\right),
$$

where $\tilde{M}_{\pi}$ denotes the explicit $M_{\pi}$-dependence from the pion propagator in the OPE potential. Without going into the details of the individual terms given here, we write the variation of the two-alpha cluster energy around the physical point as

$$
\begin{aligned}
\left.\frac{\partial E_{\alpha \alpha}}{\partial M_{\pi}}\right|_{M_{\pi}^{\mathrm{ph}}}= & \left.\frac{\partial E_{\alpha \alpha}}{\partial \tilde{M}_{\pi}}\right|_{M_{\pi}^{\mathrm{ph}}}+\left.x_{1} \frac{\partial E_{\alpha \alpha}}{\partial m_{N}}\right|_{m_{N}^{\mathrm{ph}}} \\
& +\left.x_{2} \frac{\partial E_{\alpha \alpha}}{\partial \tilde{g}_{\pi N}}\right|_{\tilde{g}_{\pi N}^{\mathrm{ph}}}+\left.x_{3} \frac{\partial E_{\alpha \alpha}}{\partial C_{0}}\right|_{C_{0}^{\mathrm{ph}}}+\left.x_{4} \frac{\partial E_{\alpha \alpha}}{\partial C_{I}}\right|_{C_{I}^{\mathrm{ph}}},
\end{aligned}
$$

\footnotetext{
${ }^{2}$ Note that throughout we do not consider variations of the strange quark mass $m_{s}$, as these are expected to be very small. Hence $m_{s}$ is simply kept at its physical value.
} 
where

$$
x_{1}=\left.\frac{\partial m_{N}}{\partial M_{\pi}}\right|_{M_{\pi}^{\mathrm{ph}}}, x_{2}=\left.\frac{\partial \tilde{g}_{\pi N}}{\partial M_{\pi}}\right|_{M_{\pi}^{\mathrm{ph}}}, x_{3}=\left.\frac{\partial C_{0}}{\partial M_{\pi}}\right|_{M_{\pi}^{\mathrm{ph}}}, x_{4}=\left.\frac{\partial C_{I}}{\partial M_{\pi}}\right|_{M_{\pi}^{\mathrm{ph}}} .
$$

The partial derivatives in eq. (3.2) are computed using the auxiliary field quantum Monte Carlo (AFQMC) method [29], see section 6. To obtain an accurate and modelindependent description of the $M_{\pi}$-dependence of the LO nuclear interaction, we will use the most recent knowledge from chiral perturbation theory and lattice QCD simulations to determine the quantities in eq. (3.3).

The partial derivative $\partial E_{\alpha \alpha} / \partial \tilde{M}_{\pi}$ in eq. (3.2) is computed by introducing a small change in the pion mass in the OPE of the nuclear Hamiltonian, $H\left(\tilde{M}_{\pi}\right) \rightarrow H\left(\tilde{M}_{\pi}+\Delta \tilde{M}_{\pi}\right)$, which corresponds to a perturbative shift in the energy, $\Delta E_{\alpha \alpha}\left(\tilde{M}_{\pi}\right)$. In our calculations, the pion masses are shifted by $\Delta \tilde{M}_{\pi}=4.59 \mathrm{MeV}$, which equals to the empirical mass difference between the neutral and charged pions. Therefore, the partial derivative $\partial E_{\alpha \alpha} / \partial \tilde{M}_{\pi}$ is defined as

$$
\left.\frac{\partial E_{\alpha \alpha}}{\partial \tilde{M}_{\pi}}\right|_{M_{\pi}^{\mathrm{ph}}}=\frac{\Delta E_{\alpha \alpha}\left(\tilde{M}_{\pi}\right)}{\Delta \tilde{M}_{\pi}} .
$$

In what follows, we will also use the so-called $K$-factors. These are defined via

$$
K_{X}^{i}=\left.\frac{y}{X} \frac{\partial X}{\partial y}\right|_{y^{\mathrm{ph}}},
$$

where $X$ is an observable and the superscript $i=\{q, \pi, \alpha\}$ denotes the quantity $y=\left\{m_{q}, M_{\pi}, \alpha_{\mathrm{EM}}\right\}$, such that, e.g., $K_{X}^{q}$ measures the sensitivity of $X$ to changes in the light quark mass $m_{q}$. For more detailed discussion on these quantities, see, e.g., ref. [19].

The parameter $x_{1}$ can be determined from the pion-nucleon sigma term,

$$
\sigma_{\pi N}=\langle N| \hat{m}\left(\bar{u} u+\bar{d} d|N\rangle=M_{\pi}^{2} \frac{\partial m_{N}}{\partial M_{\pi}^{2}},\right.
$$

i.e. the quark mass dependence of the nucleon mass, via

$$
x_{1}=\left.\frac{\partial m_{N}}{\partial M_{\pi}}\right|_{M_{\pi}^{\mathrm{ph}}}=\frac{2}{M_{\pi}} \sigma_{\pi N} .
$$

The most recent and precise values for $\sigma_{\pi N}$ are from the Roy-Steiner-equation analyses of pion-nucleon scattering $[32,34]$. In the calculation with the inclusion of pionic hydrogen and deuterium data, the reported value is $\sigma_{\pi N}=(59.1 \pm 3.5) \mathrm{MeV}$, and in the calculation using only the pion-nucleon scattering data the value is $\sigma_{\pi N}=(58.1 \pm 5) \mathrm{MeV}$. In this study we use the value of ref. [32] and the uncertainty of ref. [34], which gives

$$
x_{1}=0.84(7) .
$$

The parameter $x_{2}$ in eq. (3.3) represents the dependence of the strength of the OPE potential and is given as,

$$
x_{2}=\left.\frac{1}{2 F_{\pi}} \frac{\partial g_{A}}{\partial M_{\pi}}\right|_{M_{\pi}^{\mathrm{ph}}}-\left.\frac{g_{A}}{2 F_{\pi}^{2}} \frac{\partial F_{\pi}}{\partial M_{\pi}}\right|_{M_{\pi}^{\mathrm{ph}}} .
$$


For the dependence of $F_{\pi}$ on $M_{\pi}$ we use the results reported in ref. [19]

$$
\left.\frac{\partial F_{\pi}}{\partial M_{\pi}}\right|_{M_{\pi}^{\mathrm{ph}}}=\frac{F_{\pi}}{M_{\pi}} \frac{K_{F_{\pi}}^{q}}{K_{M_{\pi}}^{q}}=0.066(16) .
$$

The $M_{\pi}$-dependence of the nucleon axial-vector coupling $g_{A}$ is obtained from the analysis of the high-precision lattice QCD calculations [35]. We define

$$
\frac{\partial g_{A}}{\partial M_{\pi}}=\frac{\partial g_{A}}{\partial M^{*}} \frac{\partial M^{*}}{\partial M_{\pi}}
$$

where

$$
\frac{\partial M^{*}}{\partial M_{\pi}}=\frac{\partial}{\partial M_{\pi}}\left(\frac{M_{\pi}}{4 \pi F_{\pi}}\right)=\frac{1}{4 \pi F_{\pi}}\left(1-\frac{M_{\pi}}{F_{\pi}} \frac{\partial F_{\pi}}{\partial M_{\pi}}\right)=0.078(2) \text { l.u. }
$$

where l.u. stands for lattice units and $\partial g_{A} /\left.\partial M^{*}\right|_{M_{\pi}^{\text {ph }}}=-0.08(24)$. In eq. (3.12) we use the isospin-averaged pion mass $M_{\pi}=138.03 \mathrm{MeV}$. Putting pieces together, we have

$$
\frac{\partial g_{A}}{\partial M_{\pi}}=-0.006(19) \text { l.u. }
$$

which gives

$$
x_{2}=-0.053(16) \text { l.u. }
$$

So far we have discussed the quantities $x_{1}$ and $x_{2}$ which control the $M_{\pi}$-dependence of the pion and nucleon properties as well as their interactions. As has been shown, we obtained a model-independent description of these quantities utilizing the results from CHPT calculations and the data from high-precision lattice QCD. Now we turn to the discussion of the quantities $x_{3}$ and $x_{4}$ which are controlling the implicit $M_{\pi}$-dependence of the LECs of the short-range NN interactions, $C_{0}\left(M_{\pi}\right)$ and $C_{I}\left(M_{\pi}\right)$. Since the coupling constants $C_{0}$ and $C_{I}$ are adjusted to reproduce the NN scattering phase shifts in the ${ }^{1} S_{0}$ and ${ }^{3} S_{1}$ partial waves, it is much more convenient to express the $x_{3}$ and $x_{4}$ quantities in terms of the inverse singlet $(s)$ and triplet $(t)$ NN scattering lengths,

$$
\bar{A}_{s}=\left.\frac{\partial a_{s}^{-1}}{\partial M_{\pi}}\right|_{M_{\pi}^{\mathrm{ph}}}, \quad \bar{A}_{t}=\left.\frac{\partial a_{t}^{-1}}{\partial M_{\pi}}\right|_{M_{\pi}^{\mathrm{ph}}} .
$$

To obtain the desired expressions, we adopt the analysis of ref. [10], which employs the Lüscher finite volume formula to relate the spectrum of the NN system in a cubic periodic box to the NN scattering parameters,

$$
\begin{aligned}
& x_{3}=0.04847+0.06713 x_{1}-0.25101 x_{2}-0.37652 \bar{A}_{s}-0.20467 \bar{A}_{t}, \\
& x_{4}=0.04990-0.00190 x_{1}-0.01253 x_{2}-0.12551 \bar{A}_{s}+0.20467 \bar{A}_{t} .
\end{aligned}
$$

We further use the analysis of ref. [11], which determines $\bar{A}_{s}$ and $\bar{A}_{t}$ from the most recent available lattice QCD data, see appendix A for details:

$$
\bar{A}_{s}=0.54(24), \quad \bar{A}_{t}=0.33(16) .
$$


Finally, using the results given in eq. (3.18) with eq. (3.17), we get,

$$
x_{3}=-0.153(96), \quad x_{4}=0.049(46) .
$$

In what follows, we will use the values for $\bar{A}_{s, t}$ collected in eq. (3.18), noting that these are still affected by sizeable uncertainties (for a more detailed discussion, see ref. [11]). This can only be sharpened by more precise lattice QCD calculation at lower pion (quark) masses.

\section{Dependence of the nuclear Hamiltonian on the fine-structure constant}

First, we must briefly discuss how the electromagnetic interaction is included in our scheme. This requires a multi-step procedure. In a first step, we consider 8 nucleons (4 protons and 4 neutrons) in a box of $V \simeq(16 \mathrm{fm})^{3}$, from which two $\alpha$ clusters are formed. Here, the EM interaction is included using the standard power counting, see e.g. [36]. In this counting, the EM interactions start to contribute at NLO. To account for the infinitely-ranged Coulomb interaction between these two clusters with charge $Z=2$ each, we employ a second box of about $V \simeq(100 \mathrm{fm})^{3}$, which is far beyond the range of the strong interactions. Within this box, a spherical wall with a radius of about $35 \mathrm{fm}$ is placed subject to Coulomb boundary conditions. This allows for an exact treatment of the long-range Coulomb forces with the two $\alpha$ particles. For details on this procedure, we refer to refs. [15, 16].

Now, we are in the position to consider the second term on the right-hand side of eq. (2.1), which is the $\alpha_{\mathrm{EM}}$-dependence of $\alpha$ - $\alpha$ scattering. To study the $\alpha_{\mathrm{EM}}$-dependence of $\alpha$ - $\alpha$ scattering we compute the shifts $\Delta E_{\alpha \alpha}\left(\alpha_{\mathrm{EM}}\right)$ and $\Delta E_{\alpha \alpha}\left(c_{p p}\right)$. The former is the variation of two-alpha cluster energy due to the long-range Coulomb interaction, and the latter is the variation of two-alpha cluster energy due to a derivative-less proton-proton contact operator. This operator arises from the fact that the Coulomb interaction on the lattice becomes singular when two protons are on the same lattice site which requires a special treatment. Thus, a regularized version of the Coulomb interaction on the lattice is employed, and the coefficient of the proton-proton contact operator, $c_{p p}$, is determined from the proton-proton phase shifts on the lattice. The energy shift becomes,

$$
Q_{\mathrm{EM}}\left(E_{\alpha \alpha}\right)=\Delta E_{\alpha \alpha}\left(\alpha_{\mathrm{EM}}\right)+x_{p p} \Delta E_{\alpha \alpha}\left(c_{p p}\right)
$$

where $x_{p p}$ is the relative strength of the proton-proton contact term caused by the regularization of the Coulomb force. The coefficient $x_{p p}$ is computed using the data for ${ }^{4} \mathrm{He}[10]$,

$$
x_{p p}=0.39(5)
$$

Finally, the partial derivative in eq. (2.1) can be written as

$$
\left.\frac{\partial E_{\alpha \alpha}}{\partial \alpha_{\mathrm{EM}}}\right|_{\alpha_{\mathrm{EM}}^{\mathrm{ph}}} \simeq \frac{\partial Q_{\mathrm{EM}}\left(E_{\alpha \alpha}\right)}{\partial \alpha_{\mathrm{EM}}^{\mathrm{ph}}} .
$$




\section{Theta-dependence of alpha-alpha scattering}

We also strive to assess the $\theta$-dependence of $\alpha$ - $\alpha$ scattering. To that end, one might be tempted to again employ a linear variation $\propto \delta \theta$ around the physical value of $\theta^{\mathrm{ph}}$, similar to what we do in the case of the $M_{\pi}$-variation and the $\alpha_{\mathrm{EM}}$-dependence of $E_{\alpha \alpha}$, see eq. (2.1). However, it is well known that "small" variations of $\theta$ do not lead to drastic changes of nuclear physics [21, 22] and after all it is interesting in its own right to assess what is happening when $\theta$ approaches a value of, say, $\mathcal{O}(1)$. In this regime, a simple linear variation clearly would not be applicable any longer.

There is, however, a way to circumvent such a direct calculation of the $\theta$-dependence, which is based on the observation that in a first approximation any source of $\theta$-dependence of $E_{\alpha \alpha}$ can be traced back to the $\theta$-dependence of $M_{\pi}$, which in the isospin limit is given by $[37]^{3}$

$$
M_{\pi}^{2}(\theta)=2 B_{0} \hat{m} \cos \frac{\theta}{2},|\theta|<\pi .
$$

Assuming this approximation is valid, the present calculation of the $M_{\pi}$-dependence of $E_{\alpha \alpha}$ within a range of $\left|\delta M_{\pi}\right| \lesssim 0.1 M_{\pi}^{\mathrm{ph}}$ can directly be translated into an assessment of the $\theta$-dependence in a corresponding range of $|\delta \theta| \lesssim 1$.

It is not obvious that this approximation is legitimate, as $M_{\pi}$ and $\theta$ in CHPT are in principle independent parameters, but it can be justified as follows: removing the QCD $\theta$-term by a suitable choice of an axial U(1) transformation adds a complex phase

$$
\mathcal{M} \rightarrow e^{\mathrm{i} \frac{\theta}{2}} \mathcal{M}=: \mathcal{M}_{\theta}
$$

to the quark mass matrix. This $\theta$-dependent matrix enters chiral perturbation theory via the matrix $\chi_{\theta}=2 B_{0} \mathcal{M}_{\theta}$, which in the isospin symmetric case is simply given by

$$
\chi_{\theta}=\left(M_{\pi}^{2}(\theta)+\mathrm{i} 2 B_{0} \hat{m} \sin \frac{\theta}{2}\right) \mathbb{1} .
$$

Hence, inserting this expression into a given chiral Lagrangian of any order will produce terms that are either proportional to (some power of) $M_{\pi}(\theta)$, or proportional to (some power of) $\sin \theta / 2$ (or both). While the latter are naturally absent in chiral perturbation theory at $\theta=0$, the former simply leads to the known $M_{\pi}$-dependence of quantities such as $m_{N}, \tilde{g}_{\pi N}$, or couplings of nucleons to two or more pions.

As it turns out, at NLO, which is the maximal order we are considering here, the only term $\propto \sin \theta / 2$ that might alter any of the involved quantities, in particular $m_{N}$ or $\tilde{g}_{\pi N}$, comes from the NLO pion-nucleon Lagrangian [40]

$$
\mathcal{L}_{\pi N}^{(2)}=c_{5} \bar{N}\left(\chi_{+}-\frac{1}{2} \operatorname{Tr} \chi_{+}\right) N+\ldots,
$$

where $c_{5}$ is another LEC, the ellipses represent other NLO terms that are of no interest here, and

$$
\chi_{+}=u^{\dagger} \chi u^{\dagger}+u \chi^{\dagger} u
$$

\footnotetext{
${ }^{3}$ The physics at $\theta=\pi$ is a bit more involved, see, e.g., [38, 39].
} 
with $u$ carrying the pion fields. This term adds a contribution to the pion-nucleon coupling that is explicitly $\theta$-dependent, but it can be shown that its actual numerical impact is so small $(\lesssim 1-2 \%)[21,41]$ that it can safely be neglected. The smallness of these effects can directly be traced back to the suppression of the LEC $c_{5}=(-0.09 \pm 0.01) \mathrm{GeV}^{-1}$ as it parameterizes the leading isospin-breaking effects in the pion-nucleon sector [40]. This means that as long as we stick to a calculation that is of NLO at most, any non-negligible $\theta$-dependence indeed only appears implicitly in form of $M_{\pi}(\theta)$ as a consequence of the first term of eq. (5.3).

Thus, our approach here is to not perform a separate calculation for assessing the $\theta$-dependence of $\alpha$ - $\alpha$ scattering, but to simply use the results of the $M_{\pi}$-dependence analysis and map them onto the $\theta$-dependence using eq. (5.1).

\section{Adiabatic projection method and auxiliary field quantum Monte Carlo simulations}

The adiabatic projection method is a general framework to construct a low-energy effective theory for clusters. The adiabatic projection in Euclidean time gives a systematically improvable description of the low-lying scattering cluster states and in the limit of large Euclidean projection time the description becomes exact. The details of the method can be found in refs. $[12,16]$. The method starts with defining Slater-determinant of two-alpha initial cluster states $|\vec{R}\rangle$ parameterized by the relative spatial separation between the clusters on a periodic cubic lattice with a box size $L$,

$$
|\vec{R}\rangle=\sum_{\vec{r}}|\vec{r}+\vec{R}\rangle_{1} \otimes|\vec{r}\rangle_{2}
$$

To perform the calculations efficiently, we project the initial states onto spherical harmonics with angular momentum quantum numbers $\ell$ and $\ell_{z}$. To that end, we bin the cubic lattice points $\left\langle n_{x}, n_{y}, n_{z}\right\rangle$ with the same distance $|\vec{R}|=\sqrt{n_{x}^{2}+n_{y}^{2}+n_{z}^{2}}$ by weighting with spherical harmonics $Y_{\ell, \ell_{z}}(\hat{R})$,

$$
|R\rangle^{\ell, \ell_{z}}=\sum_{\vec{r}} Y_{\ell, \ell_{z}}\left(\hat{R}^{\prime}\right) \delta_{\vec{R},\left|\vec{R}^{\prime}\right|}|\vec{R}\rangle
$$

Here, $\ell$ and $\ell_{z}$ are not exactly good quantum numbers, see the discussion in ref. [43]. Since the initial cluster states are not necessarily orthonormal, we define the orthonormal initial cluster states

$$
|\mathcal{R}\rangle^{\ell, \ell_{z}}=\sum_{R^{\prime}}\left|R^{\prime}\right\rangle^{\ell, \ell_{z}}\left[N_{0}^{-1 / 2}\right]_{R^{\prime}, R}^{\ell, \ell_{z}}
$$

where $\left[N_{0}^{-1}\right]_{R^{\prime}, R}^{\ell, \ell z}$ is the norm matrix defined as

$$
\left[N_{0}^{-1}\right]_{R^{\prime}, R}^{\ell, \ell_{z}}={ }^{\ell, \ell_{z}}\left\langle R^{\prime} \mid R\right\rangle^{\ell, \ell_{z}}
$$


In the next step, the initial cluster states are evolved in Euclidean time by means of multiplying by powers of the leading order (LO) transfer matrix to form dressed cluster states,

$$
|R\rangle_{n_{t}}^{\ell, \ell_{z}}=M_{\mathrm{LO}}^{n_{t}}|\mathcal{R}\rangle^{\ell, \ell_{z}}
$$

This procedure, by design, incorporates all the induced deformations and polarizations of the alpha clusters due to the microscopic interaction and it gives the true low-lying cluster states of the transfer matrix $M_{\mathrm{LO}}$. In general the dressed cluster states are not orthonormal, thus for further calculations we use the following form of the dressed cluster states,

$$
|\mathcal{R}\rangle_{n_{t}}^{\ell, \ell_{z}}=\sum_{R^{\prime}}\left|R^{\prime}\right\rangle_{n_{t}}^{\ell, \ell_{z}}\left[N_{L_{t}}^{-1 / 2}\right]_{R^{\prime}, R}^{\ell, \ell_{z}}
$$

where $\left[N_{L_{t}}^{-1 / 2}\right]_{R, R^{\prime}}^{\ell, \ell_{z}}$ is the norm matrix at Euclidean time $L_{t}=2 \times n_{t}$. Finally, we define the radial adiabatic transfer matrix at $\mathrm{LO}$ as,

$$
\left[M_{\mathrm{LO}, L_{t}}^{a}\right]_{R, R^{\prime}}^{\ell, \ell_{z}}={ }_{n_{t}}^{\ell, \ell_{z}}\left\langle\mathcal{R}\left|M_{\mathrm{LO}}\right| \mathcal{R}^{\prime}\right\rangle_{n_{t}}^{\ell, \ell_{z}} .
$$

In our calculation the higher-order $(\mathrm{HO})$ interactions are treated using first-order perturbation theory, thus we include the perturbative contributions from NLO, next-tonext-to-leading order (NNLO), isospin-breaking (IB), and Coulomb interactions (EM) to the leading-order radial adiabatic transfer matrix order-by-order in perturbation theory. Therefore, we define the radial adiabatic transfer matrix at a given higher order in a closed form as

$$
\left[M_{\mathrm{HO}, L_{t}}^{a}\right]_{R, R^{\prime}}^{\ell, \ell_{z}}={ }_{n_{t}}^{\ell, \ell_{z}}\left\langle\mathcal{R}\left|M_{\mathrm{LO}}\right| \mathcal{R}^{\prime}\right\rangle_{n_{t}}^{\ell, \ell_{z}}-\alpha_{t} \stackrel{\ell, \ell_{z}}{n_{t}}\left\langle\mathcal{R}\left|: V_{\mathrm{HO}} M_{\mathrm{LO}}:\right| \mathcal{R}^{\prime}\right\rangle_{n_{t}}^{\ell, \ell_{z}}
$$

where $\alpha_{t}=a_{t} / a$ is the ratio of the temporal and the spatial lattice spacings, and $V_{\mathrm{HO}}$ is the higher-potential at the order of interest. The colons : ... : denote normal ordering, which means that we reorder the creation and annihilation operators inside the colons and we move the creation operators to the left of the all annihilation operators with the appropriate number of anti-commutation minus signs.

So far we have discussed the adiabatic projection method for the chiral EFT Hamiltonian. Now we turn to the main interest of this paper, which is to construct the two-cluster matrix elements of the partial derivatives given in eq. (2.1). Due to the fact that we study the effects of small variations in the fundamental constants of nature on $\alpha$ - $\alpha$ scattering, the partial derivatives in eq. (2.1) are treated in a similar manner as the higher-order corrections,

$$
\begin{aligned}
{\left[M_{\mathrm{HO}, L_{t}}^{a, y}\right]_{R, R^{\prime}}^{\ell, \ell_{z}}=} & { }_{n_{t}}^{\ell, \ell_{z}}\left\langle\mathcal{R}\left|M_{\mathrm{LO}}\right| \mathcal{R}^{\prime}\right\rangle_{n_{t}}^{\ell, \ell_{z}} \\
& -\alpha_{t} \stackrel{{ }_{n}, \ell_{z}}{n_{t}}\left\langle\mathcal{R}\left|: V_{\mathrm{HO}} M_{\mathrm{LO}}:\right| \mathcal{R}^{\prime}\right\rangle_{n_{t}}^{\ell, \ell_{z}} \\
& -\alpha_{t}{ }_{n_{t}}^{\ell, \ell_{z}}\left\langle\mathcal{R}\left|: \frac{\partial E_{\alpha \alpha}}{\partial y}\right|_{y_{\mathrm{ph}}} M_{\mathrm{LO}}: \mid \mathcal{R}^{\prime}\right\rangle_{n_{t}}^{\ell, \ell_{z}} \delta y,
\end{aligned}
$$

we use the superscript $y$ for the observables $M_{\pi}, \alpha_{\mathrm{EM}}$ and, in principle, $\theta$. However, as discussed in section 5 , we will not perform explicit differentiations with respect to $\theta$. 
The two-cluster matrix elements of the LO transfer matrix, the higher order corrections, and the partial derivatives are computed by means of the auxiliary field quantum Monte Carlo (AFQMC) method. The non-perturbative quantum Monte Carlo simulations are performed using the neutral pion mass $M_{\pi}$ and the isospin symmetry breaking effects are incorporated perturbatively. The calculation of the radial adiabatic transfer matrices in eqs. (6.7), (6.8) and (6.9) is divided into two separate parts. In the first part of the calculation, we perform the AFQMC simulation for the system of $A=8$ nucleons (4 protons and 4 neutrons) to construct the radial adiabatic transfer matrices for two interacting $\alpha$ clusters. Due to the computational cost associated with such simulations, this is done on a periodic cubic lattice of length $L$ which is not too large to prevent us from computing the matrices accurately but is not too small so that the length $L / 2$ is much larger than the range of the interaction, $R \sim 1 / M_{\pi} \simeq 1.4 \mathrm{fm}$. In the second part of the calculation, the AFQMC simulations are performed for the system with $A=4$ nucleons, and these simulations are done on a periodic cubic lattice of larger length due to the less computational demand. This single $\alpha$ cluster adiabatic matrix is used to construct the radial adiabatic transfer matrices for non-interacting two $\alpha$ clusters. Finally, we connect the radial adiabatic transfer matrices of interacting $\alpha$ clusters with the radial adiabatic transfer matrices of non-interacting $\alpha$ clusters in the asymptotic region to extend the radial transfer matrix of interacting $\alpha$ clusters to a larger volume. The aforementioned two-part approach was studied extensively for nucleon-deuteron systems in ref. [16], and it was found that the systematic errors due to extension of the radial transfer matrix are negligible.

The first $a b$ initio calculation of $\alpha$ - $\alpha$ scattering was performed in ref. [15] using the same chiral Hamiltonian as adopted in this paper. However, in this paper we employ developments in the adiabatic projection method from refs. [16, 23, 42]. As discussed above, the first step of the adiabatic projection method is to define the initial cluster states, and on a periodic cubic lattice of length $L$ the total number of initial cluster states parameterized by the relative spatial separation is $N_{\vec{R}}=3 L^{2} / 4$. In ref. [12] it was shown that it is not required to use every possible cluster state when we are interested in only a few low-lying energies of the system of interest. Therefore, for simulating computationally demanding systems it is advantageous to construct a radial adiabatic transfer matrix defined in the subspace that is spanned by $N_{\vec{R}}<3 L^{2} / 4$ cluster separation states. Following these findings, in ref. [15] the radial adiabatic transfer matrix for non-interacting two-alpha clusters was constructed in a smaller subspace of the two-cluster state space. In this paper, taking advantage of powerful computational resources we perform our simulations using every possible cluster state and construct the radial adiabatic transfer matrices in full space of the two-cluster state space.

\section{Extracting scattering phase shifts from the adiabatic matrices}

What was discussed in the previous section was the first part of the adiabatic projection method, which is constructing the adiabatic transfer matrix for the two clusters. The second part of the method is to extract the scattering or reaction parameters for the two clusters. In the previous section, by projecting the initial cluster states onto spherical harmonics 
with angular momentum quantum numbers $\ell$ and $\ell_{z}$, we constructed the adiabatic transfer matrix in radial coordinates, which provides a significant improvement in the computational scaling [16]. Since our adiabatic transfer matrices are defined in radial coordinates, the best approach to be used to calculate the scattering parameters is the so-called spherical wall method [43-45].

In the spherical wall method we employ a hard boundary wall condition at $r=R_{\text {wall }}$, which is the relative separation distance between two clusters in the asymptotic region. In general, the spherical wall method is used to remove the periodic boundary effects inherited from the cubic lattice and the artifacts due to the periodic boundary condition. However, in our calculations these effects are already eliminated since we construct the adiabatic transfer matrices in radial coordinates as explained in section 6. After imposing the spherical hard wall to the radial adiabatic transfer matrices, we solve the Schrödinger equation of the system and obtain the spherical scattering wave functions as well as the spectrum. In principle, due to the imposed spherical hard wall one expects that the spherical wave functions die out at $R_{\text {wall }}$, however, as a result of non-zero spatial lattice spacing the spherical wave functions vanish at $R_{\text {wall }}^{\prime}=R_{\text {wall }}+\varepsilon_{R}$, where $\varepsilon_{R}$ is the correction on the precise radius of the spherical wall and is defined as $\left|\varepsilon_{R}\right|<a / 2$.

The total wave function of a two-cluster system is decomposed into the radial part $R_{\ell}^{(p)}(r)$ and the spherical harmonics $Y_{\ell, \ell_{z}}(\hat{r})$,

$$
\Psi(\vec{r})=R_{\ell}^{(p)}(r) Y_{\ell, \ell_{z}}(\hat{r})
$$

where $r$ is the relative spatial separation of the clusters and $p$ is the relative momentum. The radial wave function in the asymptotic region is given by

$$
R_{\ell}^{(p)}(r)=N_{\ell}(p)\left[\cos \delta_{\ell}(p) F_{\ell}(p r)+\sin \delta_{\ell}(p) G_{\ell}(p r)\right]
$$

where $N_{\ell}(p)$ is an overall normalization coefficient, and $F_{\ell}\left(G_{\ell}\right)$ is the regular (irregular) Coulomb wave function.

The relative momentum $p$ is calculated from the spectrum of the radial adiabatic transfer matrices and the dispersion relation of the two-cluster system given by,

$$
E=c_{0} \frac{p^{2}}{2 \mu}+c_{1} p^{4}+c_{2} p^{6}+\ldots
$$

where $\mu=m_{\alpha} / 2$ is the reduced mass of the two-cluster system, $m_{\alpha}$ the mass of the $\alpha$ particle, and the coefficients $c_{i}$ are determined by fitting eq. (7.3) to the lattice dispersion relation. We determine the correction $\varepsilon_{R}$ from the roots of the regular Coulomb wave function with the relative momentum of the non-interacting two-cluster system, $p_{0}$, around $R_{\text {wall }}$. Finally, we use the corrected radius of the spherical hard wall, $R_{\text {wall }}^{\prime}$, and the relative momentum of the interacting two-cluster system, $p$, and solve eq. (7.2) for the scattering phase shifts,

$$
\delta_{\ell}(p)=\tan ^{-1}\left[-\frac{F_{\ell}\left(p R_{\text {wall }}^{\prime}\right)}{G_{\ell}\left(p R_{\text {wall }}^{\prime}\right)}\right]
$$





Figure 1. Left panel: S-wave $\alpha$ - $\alpha$ scattering phase shift $\delta_{0}$ versus the energy in the laboratory system, $E_{\text {lab. }}$ Right panel: D-wave $\alpha$ - $\alpha$ scattering phase shift $\delta_{2}$ versus the energy in the laboratory system, $E_{\text {lab. }}$. The blue circles and red squares represent our predictions at NLO and NNLO, respectively, while the data are given by the black crosses.

We extract the scattering phase shifts from the radial adiabatic transfer matrices with $L_{t}$ time steps and perform Euclidean time extrapolating to the limit $L_{t} \rightarrow \infty$. Details of the extrapolation fit and all associated error estimates are discussed in appendix B.

\section{Results}

\subsection{Our universe}

Here, we discuss the results for the S- and D-wave phase shifts and the effective range parameters in the S-wave as well as the resonance parameters in the $\mathrm{D}$-wave for the physical values of $M_{\pi}$ and $\alpha_{\mathrm{EM}}$ and $\theta=0$. In figure 1 , we show the S-wave phase shift $\delta_{0}$ (left panel) and the D-wave phase shift $\delta_{2}$ (right panel) at NLO and NNLO in comparison to the data [2]. Note that we do not show the LO result here, as the electromagnetic interaction is not yet included and therefore the predicted curve is far off the data (as discussed in more detail in ref. [15]). We find a marked improvement, both for the S-wave and the D-wave, as compared to the pioneering work in ref. [15], which is due to the improvements in the APM discussed in the earlier sections. We note that these are parameter-free predictions. Furthermore, the uncertainties are mostly stemming from the large Euclidean time extrapolation and these decrease when going from NLO to NNLO, as expected in a well-behaved expansion. Up to $E_{\mathrm{Lab}} \simeq 3.5 \mathrm{MeV}$, our description of the S-wave phase shift is as good as the one obtained using halo EFT in ref. [46]. We note that the uncertainties have somewhat increased as compared to ref. [15] because, as discussed in the previous section, we use a much larger subspace of the two-cluster state space, which reduces the number of configurations used for the matrix entries, resulting in a larger statistical uncertainty. This could eventually be overcome by utilizing much more HPC resources. 


\begin{tabular}{|l|c|c|c|c|c|}
\hline & \multicolumn{3}{|c|}{ S-wave } & \multicolumn{2}{c|}{ D-wave } \\
\hline & $a_{0}\left[10^{3} \mathrm{fm}\right]$ & $r_{0}[\mathrm{fm}]$ & $P_{0}\left[\mathrm{fm}^{3}\right]$ & $E_{R}[\mathrm{MeV}]$ & $\Gamma_{R}[\mathrm{MeV}]$ \\
\hline NLO & $-1.80(93)$ & $1.045(15)$ & $-2.297(156)$ & $3.05(4)$ & $2.68(23)$ \\
\hline NNLO & $-1.55(63)$ & $1.061(14)$ & $-2.277(158)$ & $2.93(5)$ & $2.00(16)$ \\
\hline empirical & $-1.65(17)$ & $1.084(11)$ & $-1.76(22)$ & $2.92(18)$ & $1.35(50)$ \\
\hline
\end{tabular}

Table 1. S-wave: the ERE parameters $a_{0}, r_{0}$ and $P_{0}$ at NLO and NNLO. D-wave: the resonance parameters $E_{R}$ and $\Gamma_{R}$ at NLO and NNLO. The empirical values from ref. [47] are also given.

Next, we discuss the S-wave ERE parameters $a_{0}, r_{0}$ and $P_{0}$ (see appendix $\mathrm{C}$ for definitions), collected in table 1 . The fit range to determine these is from $E_{\mathrm{Lab}}=1.0$ to 7.7 MeV. We see that these parameters are consistent with the empirical determinations, but they are also afflicted with sizeable uncertainties. Note that there is sensitivity to the fit range as well as to the position of the $0^{+}$resonance, the ${ }^{8} \mathrm{Be}$ ground state, as discussed in ref. [46]. In our calculation, ${ }^{8} \mathrm{Be}$ is very weakly bound. This appears to be in contradiction to the scattering lengths given in table 1 , but these values are very sensitive to the fitting range employed to extract them, see also ref. [46].

The D-wave phase shift shows a clear resonance-behaviour. Due to the large width of the resonance, the extraction of the resonance parameters (energy and width) is affected with some model-dependence. As in our earlier work, we fix the resonance energy $E_{R}$ by the maximum of $d \delta / d E$ and its width $\Gamma_{R}$ from the value of $2(d \delta / d E)^{-1}$ at $E_{R}$, see e.g. ref. [48]. The resonance parameters at NLO and NNLO are also given in table 1 . We find that the resonance parameters at NNLO are much closer to the empirical ones as compared to our earlier work.

\subsection{The Multiverse}

\subsubsection{Variations of the bound state energies}

Before considering the effect of the variations of the fundamental parameters on the $\alpha$ - $\alpha$ scattering phase shifts, we discuss briefly the variation of the various bound state energies relevant to the $3 \alpha$ process. This provides some additional information to ref. [11] that was not explicitly displayed there. Consider first pion mass variations, keeping $\alpha_{\mathrm{EM}}$ and $\theta$ at their physical values. In the left panel of figure 2 , we display the variation of the energies of ${ }^{4} \mathrm{He},{ }^{4} \mathrm{Be},{ }^{12} \mathrm{C}$ and the Hoyle state ${ }^{12} \mathrm{C}^{\star}$ as a function of the varying pion mass for positive changes in $M_{\pi}$. These energies are denoted as $E_{4}, E_{8}, E_{12}$ and $E_{12}^{\star}$, in order, see the explicit expressions in appendix D. For negative energy changes in $M_{\pi}$ these curves only differ in the sign, that is the contribution is repulsive rather than attractive as for positive shifts in the pion mass. These different energies are obviously correlated, as shown more clearly in the right panel of figure 2, where the various $K$-factors for the pertinent eight and twelve particle systems are displayed as a function of the corresponding ${ }^{4} \mathrm{He}$ $K$-factor, $K_{E_{4}}^{\pi}$, for independent variations of $\bar{A}_{s}$ and $\bar{A}_{t}$ over the range $\{-1, \ldots,+1\}$ are shown. Of course, the actual range of these parameters as given in eq. (3.18) is smaller, but these parameters might change when better results from lattice QCD will become available. 

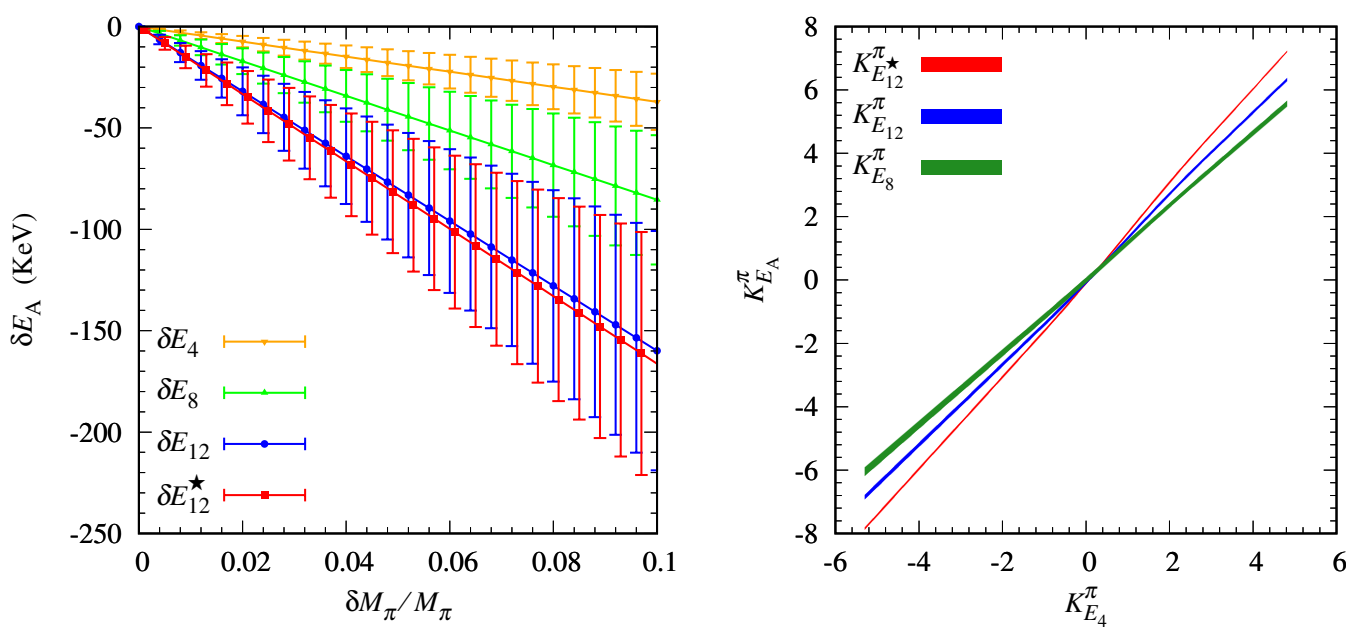

Figure 2. Left panel: variation of the ground state energy of the nuclei ${ }^{4} \mathrm{He},{ }^{4} \mathrm{Be},{ }^{12} \mathrm{C}$ and the Hoyle state ${ }^{12} \mathrm{C}^{\star}$, respectively, under variation of the pion mass (in percent). Right panel: sensitivity of the ground state energy of the nuclei ${ }^{8} \mathrm{Be},{ }^{12} \mathrm{C}$ and the Hoyle state ${ }^{12} \mathrm{C}^{\star}$, respectively, to changes in $M_{\pi}$ as a function of $K_{E_{4}}^{\pi}$ under independent variations of $\bar{A}_{s}$ and $\bar{A}_{t}$ over the range $\{-1, \ldots,+1\}$.



Figure 3. Variation of the ground state energy of the nuclei ${ }^{4} \mathrm{He},{ }^{4} \mathrm{Be},{ }^{12} \mathrm{C}$ and the Hoyle state ${ }^{12} \mathrm{C}^{\star}$, respectively, under variation of the fine-structure constant (in percent).

Note that such correlations related to the production of carbon have indeed been speculated upon earlier $[49,50]$.

Next, we consider variations of the fine-structure constant for physical pion masses and vanishing $\theta$ angle. The variations of the energies $E_{4}, E_{8}, E_{12}$ and $E_{12}^{\star}$ with varying $\alpha_{\mathrm{EM}}$ are displayed in figure 3 (for positive shifts in $\alpha_{\mathrm{EM}}$ ). Naively, one would expect the slopes of the different nuclei to scale as $Z^{2}$, that is in the ratio $1: 4: 9$ for ${ }^{4} \mathrm{He},{ }^{8} \mathrm{Be}$ and ${ }^{12} \mathrm{C}$, in order. The observed difference from this scaling is coming from the proton-proton derivativeless contact interaction. In fact, removing the contribution from this term, one finds for $\delta \alpha_{\mathrm{EM}} / \alpha_{\mathrm{EM}}=5 \%$ the following energy shifts: $\delta E_{4}=30.65(10) \mathrm{keV}, \delta E_{8}=117.5(10) \mathrm{keV}$ 
and $\delta E_{12}=283.5(10) \mathrm{keV}$, perfectly consistent with the $Z^{2}$ scaling. We note here that the results for negative shifts in $\alpha_{\mathrm{EM}}$ are of opposite sign, that is pertinent energy shifts $\delta E_{A}$ are negative.

\subsubsection{Pion mass variations of alpha-alpha scattering}

We now consider pion mass variations keeping $\alpha_{\mathrm{EM}} \simeq 1 / 137$ and $\theta \simeq 0$ fixed. In figure 4 , we display the NLO results with variations of the pion mass up to $\pm 3 \%$ (inner red bands), together with the $1 \sigma$ uncertainty of the $3 \%$ variation (outer orange bands) as well as the variations up to $5 \%$ (inner dark green bands) and the $1 \sigma$ uncertainty of the $5 \%$ variation (outer light green bands). As before, the left panel gives the S-wave $\delta_{0}$ and the right panel the $\mathrm{D}$-wave $\delta_{2}$ phase shift. The pertinent $1 \sigma$ uncertainties include all statistical and systematic errors properly propagated at this order. Consider now in more detail the S-wave. For positive pion mass shifts, there is very little effect on $\delta_{0}$, however, this is different for negative pion mass shifts. At around $\delta M_{\pi} / M_{\pi} \simeq-5 \%$, the additional repulsion unbinds the two-alpha system as seen by the phase shift starting at zero.

In the D-wave, the effects of the pion mass variation are somewhat more pronounced, as seen in the right panel of figure 4. Here, the upper (lower) part of the band refers to positive (negative) shifts in the pion mass. The pion mass variation is also reflected in the parameters of the $\mathrm{D}$-wave resonance, which for a pion mass variation of $\pm 5 \%$ are given by

$$
\begin{array}{lll}
E_{R}=2.57(6) \mathrm{MeV}, & \Gamma_{R}=1.22(21) \mathrm{MeV} & \delta M_{\pi} / M_{\pi}=+5 \%, \\
E_{R}=3.60(13) \mathrm{MeV}, & \Gamma_{R}=3.56(89) \mathrm{MeV} & \delta M_{\pi} / M_{\pi}=-5 \% .
\end{array}
$$

We now turn to the results at NNLO, showing the pertinent results for the S-wave in the left panel of figure 5 and for the $\mathrm{D}$-wave in the right panel of that figure. Consider first the Swave, where we display results for pion mass variations in the range $-7 \% \leq \delta M_{\pi} / M_{\pi} \leq 10 \%$. The critical value for $\delta M_{\pi} / M_{\pi}$, where the two-alpha system becomes unbound, is moved to $-7 \%$, where as positive changes of up to $10 \%$ do not lead to significant changes in the phase shift $\delta_{0}$. For the D-wave, we again find a larger sensitivity (see right panel of figure 5 ). This is again reflected in the resonance parameters,

$$
\begin{array}{lll}
E_{R}=2.52(15) \mathrm{MeV}, & \Gamma_{R}=0.92(33) \mathrm{MeV} & \delta M_{\pi} / M_{\pi}=+5 \%, \\
E_{R}=3.22(5) \mathrm{MeV}, & \Gamma_{R}=2.69(26) \mathrm{MeV} & \delta M_{\pi} / M_{\pi}=-5 \% .
\end{array}
$$

We note that both at NLO and NNLO, the variations of $E_{R}$ and $\Gamma_{R}$ are almost linear in the pion mass shift.

As noted, in our calculation at NNLO, the ${ }^{8}$ Be nucleus is slightly bound, which generates some of the behaviour of the phase shifts close to zero energy. To overcome this, we also consider the pion mass dependence of the S-wave effective range function $K_{0}\left(E_{\mathrm{Lab}}\right)$ as well as the one of the $\mathrm{D}$-wave effective range function $K_{2}\left(E_{\mathrm{Lab}}\right)$, as defined in appendix $\mathrm{C}$.

Let us start with the S-wave. In figure 6 , we show the pion mass variation of the S-wave effective range function with respect to the results for our Universe at NLO (left panel) and NNLO (right panel). There appears to be little effect on $K_{0}\left(E_{\text {Lab }}\right)$ at NLO, with a somewhat increased repulsion for negative pion mass shifts. More precisely, there is some 

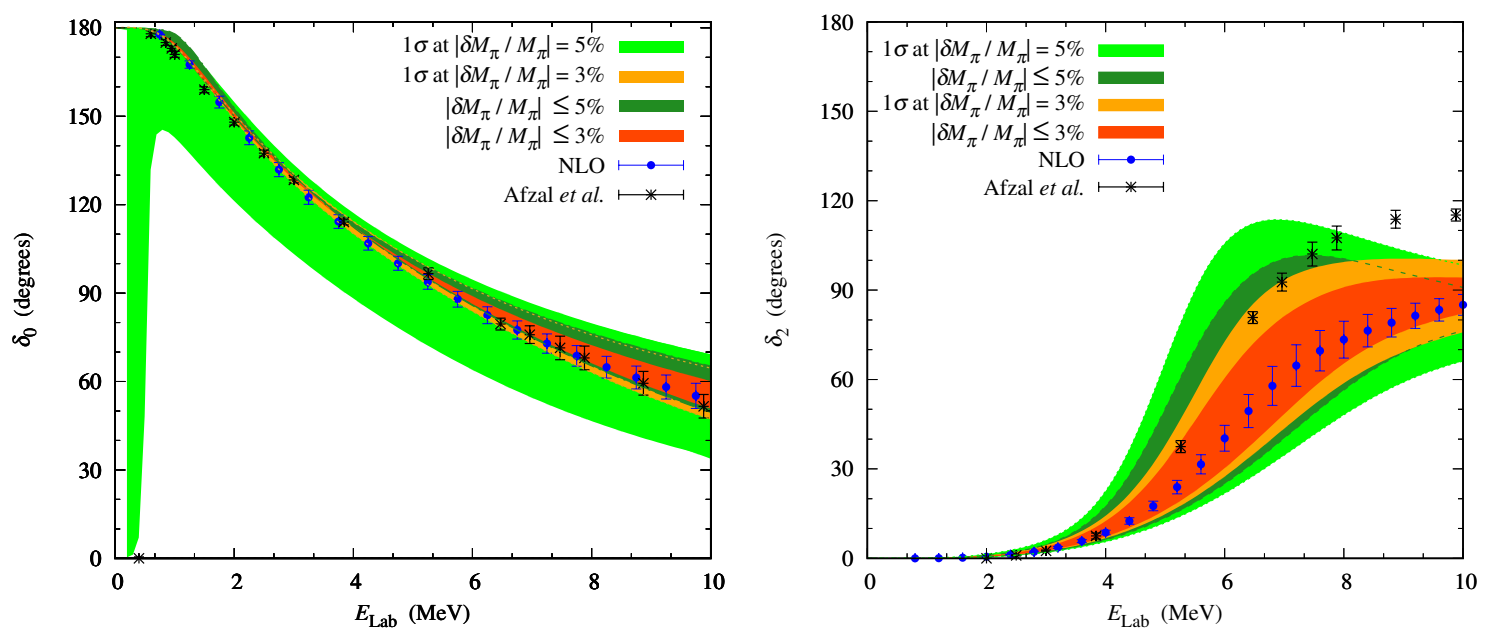

Figure 4. Pion mass dependence of the $\alpha-\alpha$ phase shifts at NLO. Left panel: S-wave phase shift $\delta_{0}$ versus the energy in the laboratory system, $E_{\text {lab }}$. Right panel: D-wave phase shift $\delta_{0}$ versus the energy in the laboratory system, $E_{\text {lab }}$. The black crosses refer to the experimental data, the blue circles are the NLO results in the limit $L_{t} \rightarrow \infty$ at $\delta M_{\pi}=0$. The red band corresponds the S-wave phase shifts with a variation in $M_{\pi}$ within $\left|\delta M_{\pi} / M_{\pi}\right| \leq 3 \%$, and the golden band refers to the errors for $\left|\delta M_{\pi} / M_{\pi}\right|=3 \%$. The dark green band corresponds to a variation in $M_{\pi}$ within $\left|\delta M_{\pi} / M_{\pi}\right| \leq 5 \%$, and the light green band refers to the errors for $\left|\delta M_{\pi} / M_{\pi}\right|=5 \%$. In the case of variation in $M_{\pi}$ by $-5 \%$ in the S-wave, due to difficulty in performing Euclidean time extrapolation at low-energies we estimate the error band from the spread in phase shifts versus the number of time steps.
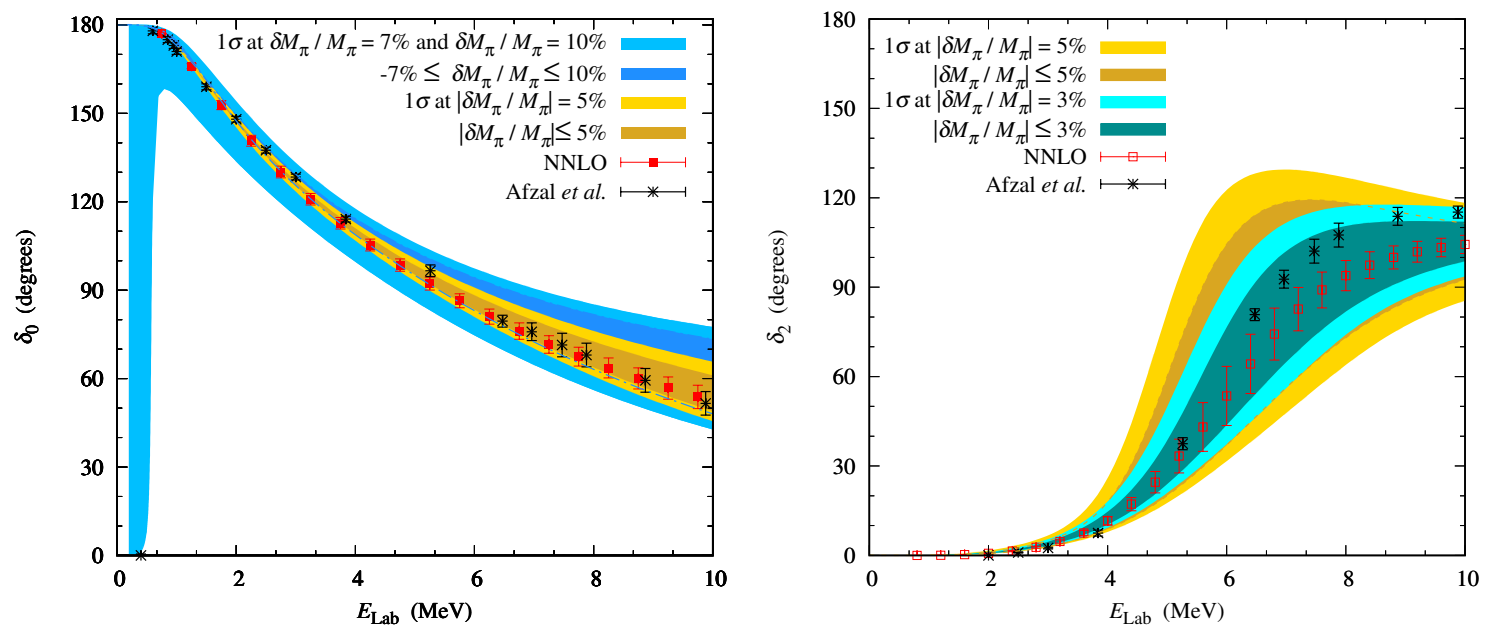

Figure 5. Pion mass dependence of the $\alpha-\alpha$ phase shifts at NNLO. Left panel: S-wave phase shift $\delta_{0}$ versus the energy in the laboratory system, $E_{\text {lab }}$. Right panel: D-wave phase shift $\delta_{0}$ versus the energy in the laboratory system, $E_{\text {lab }}$. The black crosses refer to the experimental data, the red squares are the NNLO results in the limit $L_{t} \rightarrow \infty$ at $\delta M_{\pi}=0$. The dark gold band corresponds to the S-wave phase shifts with a variation in $M_{\pi}$ within $\left|\delta M_{\pi} / M_{\pi}\right| \leq 5 \%$, and the light golden band refers to the errors for $\left|\delta M_{\pi} / M_{\pi}\right|=5 \%$. The dark blue band corresponds to a variation in $M_{\pi}$ within $-7 \% \leq \delta M_{\pi} / M_{\pi} \leq 10 \%$, and the light blue band refers to the corresponding errors. In the case of variation in $M_{\pi}$ by $-7 \%$ in the S-wave, due to the difficulty in performing a Euclidean time extrapolation at low energies, we estimate the error band from the spread in phase shifts versus the number of time steps. 

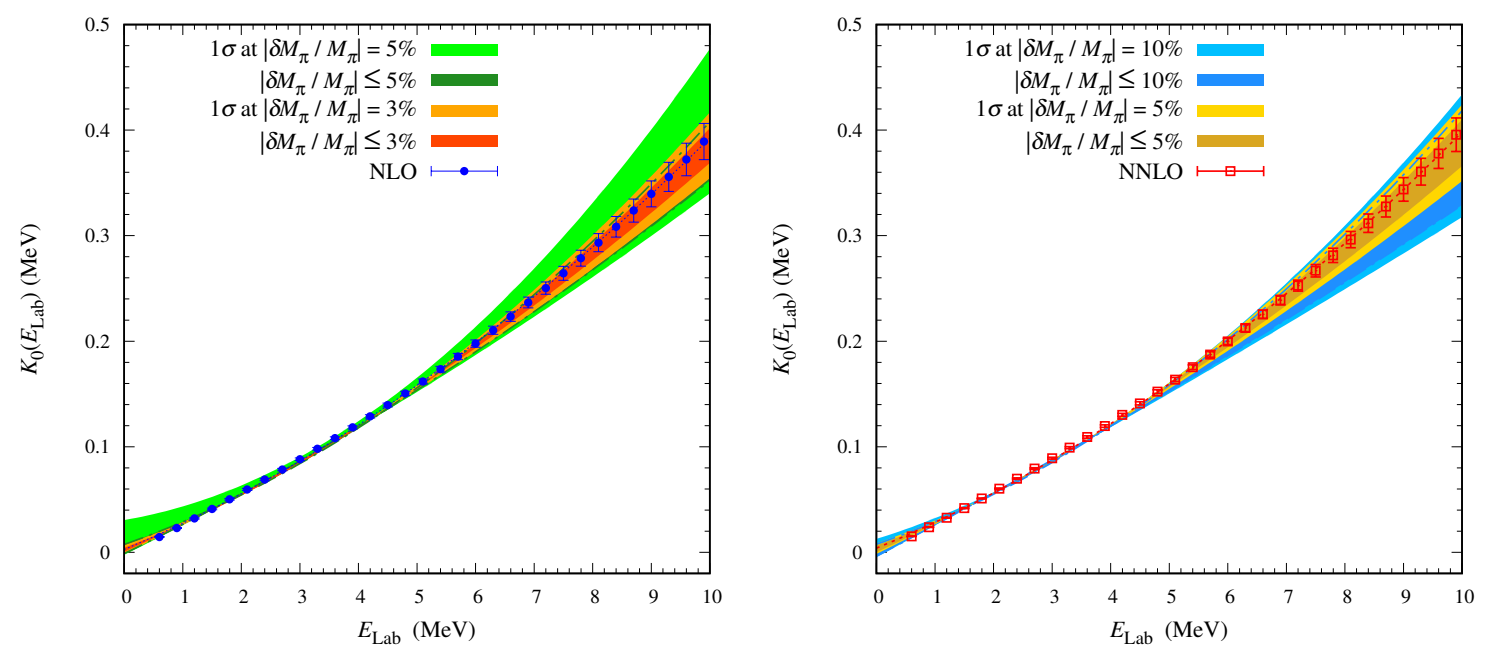

Figure 6. Pion mass dependence of the S-wave effective range function. Left panel: NLO results. The blue points refer to the results without pion mass variation, the red and dark green bands refer to changes by $\pm 5 \%$ and $\pm 10 \%$, in order, and the orange and light green bands are the corresponding $1 \sigma$ uncertainties. Right panel: NNLO results. The red squares refer to the results without pion mass variation, the golden and dark blue bands refer to changes by $\pm 5 \%$ and $\pm 10 \%$, in order, and the yellow and light blue bands are the corresponding $1 \sigma$ uncertainties.

added repulsion for negative mass shifts. This trend is also found at NNLO, with some increase in strength. We can quantify this by calculating the shift in the first parameter of the ERE, namely the inverse S-wave scattering length at NLO

$$
\frac{1}{a_{0}}=\left\{\begin{array}{lll}
-0.0017(12) & \text { for } & \delta M_{\pi} / M_{\pi}=-5 \% \\
-0.0025(3) & \text { for } & \delta M_{\pi} / M_{\pi}=-3 \% \\
-0.0019(1) & \text { for } & \delta M_{\pi} / M_{\pi}=0 \\
-0.0016(1) & \text { for } & \delta M_{\pi} / M_{\pi}=+3 \% \\
-0.0019(1) & \text { for } & \delta M_{\pi} / M_{\pi}=+5 \%
\end{array}\right.
$$

and at NNLO

$$
\frac{1}{a_{0}}=\left\{\begin{array}{lll}
+0.0011(6) & \text { for } & \delta M_{\pi} / M_{\pi}=-10 \% \\
-0.0016(1) & \text { for } & \delta M_{\pi} / M_{\pi}=-5 \% \\
-0.0014(1) & \text { for } & \delta M_{\pi} / M_{\pi}=0 \\
-0.0013(1) & \text { for } & \delta M_{\pi} / M_{\pi}=+5 \% \\
-0.0021(1) & \text { for } & \delta M_{\pi} / M_{\pi}=+10 \%
\end{array}\right.
$$

all in units of $\mathrm{MeV}$. We note that the shifts at NNLO are a bit larger than the ones at NLO, which can be traced back to the fact that there is more short-range repulsion in the NNLO interaction and thus it is less sensitive to the pion mass dependent corrections. Clearly, the NNLO calculation should be considered more reliable.

Consider now the $\mathrm{D}$-wave. In figure 7 , we show the pion mass variation of the $\mathrm{D}$-wave effective range function with respect to the results for our Universe at NLO (left panel) and NNLO (right panel). The effect on $K_{2}\left(E_{\text {Lab }}\right)$ is quite pronounced, it is smallest where the 

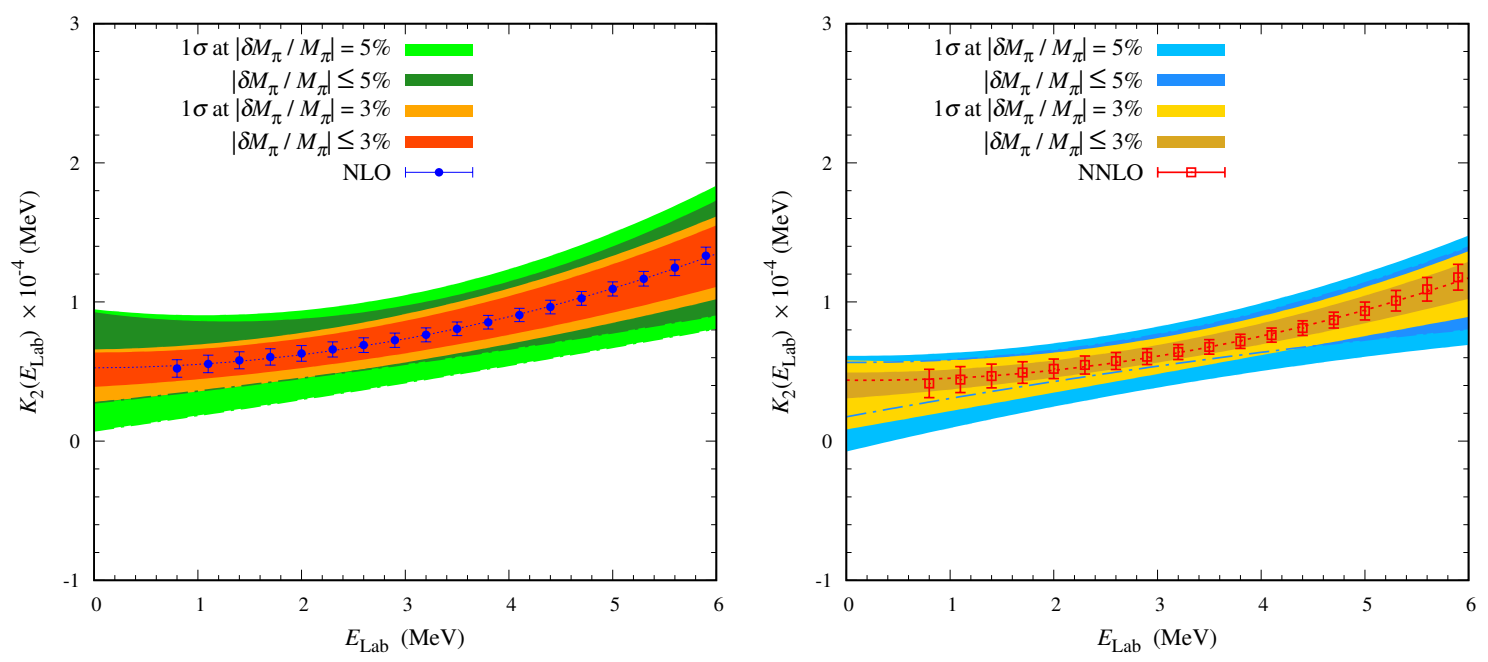

Figure 7. Pion mass dependence of the D-wave effective range function. Left panel: NLO results. The blue points refer to the results without pion mass variation, the red and dark green bands refer to changes by $\pm 5 \%$ and $\pm 10 \%$, in order, and the orange and light green bands are the corresponding $1 \sigma$ uncertainties. Right panel: NNLO results. The red squares refer to the results without pion mass variation, the golden and dark blue bands refer to changes by $\pm 5 \%$ and $\pm 10 \%$, in order, and the yellow and light blue bands are the corresponding $1 \sigma$ uncertainties.

phase shift passes through the resonance. We can quantify this by calculating the shifts in the inverse D-wave scattering length, at NLO first parameter of the ERE, namely the inverse D-wave scattering length at NLO

$$
\frac{1}{a_{2}}=\left\{\begin{array}{lll}
9.30(2) & \text { for } & \delta M_{\pi} / M_{\pi}=-5 \% \\
6.19(5) & \text { for } & \delta M_{\pi} / M_{\pi}=-3 \% \\
5.27(5) & \text { for } & \delta M_{\pi} / M_{\pi}=0 \\
3.79(6) & \text { for } & \delta M_{\pi} / M_{\pi}=+3 \% \\
2.42(12) & \text { for } & \delta M_{\pi} / M_{\pi}=+5 \%
\end{array}\right.
$$

and at NNLO

$$
\frac{1}{a_{2}}=\left\{\begin{array}{lll}
5.49(6) & \text { for } & \delta M_{\pi} / M_{\pi}=-5 \%, \\
4.95(8) & \text { for } & \delta M_{\pi} / M_{\pi}=-3 \%, \\
4.35(10) & \text { for } & \delta M_{\pi} / M_{\pi}=0, \\
3.02(4) & \text { for } & \delta M_{\pi} / M_{\pi}=+3 \%, \\
1.54(4) & \text { for } & \delta M_{\pi} / M_{\pi}=+5 \%,
\end{array}\right.
$$

all in units of $10^{-5} \mathrm{MeV}^{3}$. Again, we find somewhat reduced changes at NNLO compared to NLO.

\subsubsection{Alpha-alpha scattering with varying $\alpha_{\mathrm{EM}}$}

Here, we consider the influence of variations in the fine-structure constant on the $\alpha$ - $\alpha$ phase shifts. Despite the various sources contributing to this type of modifications as discussed in 

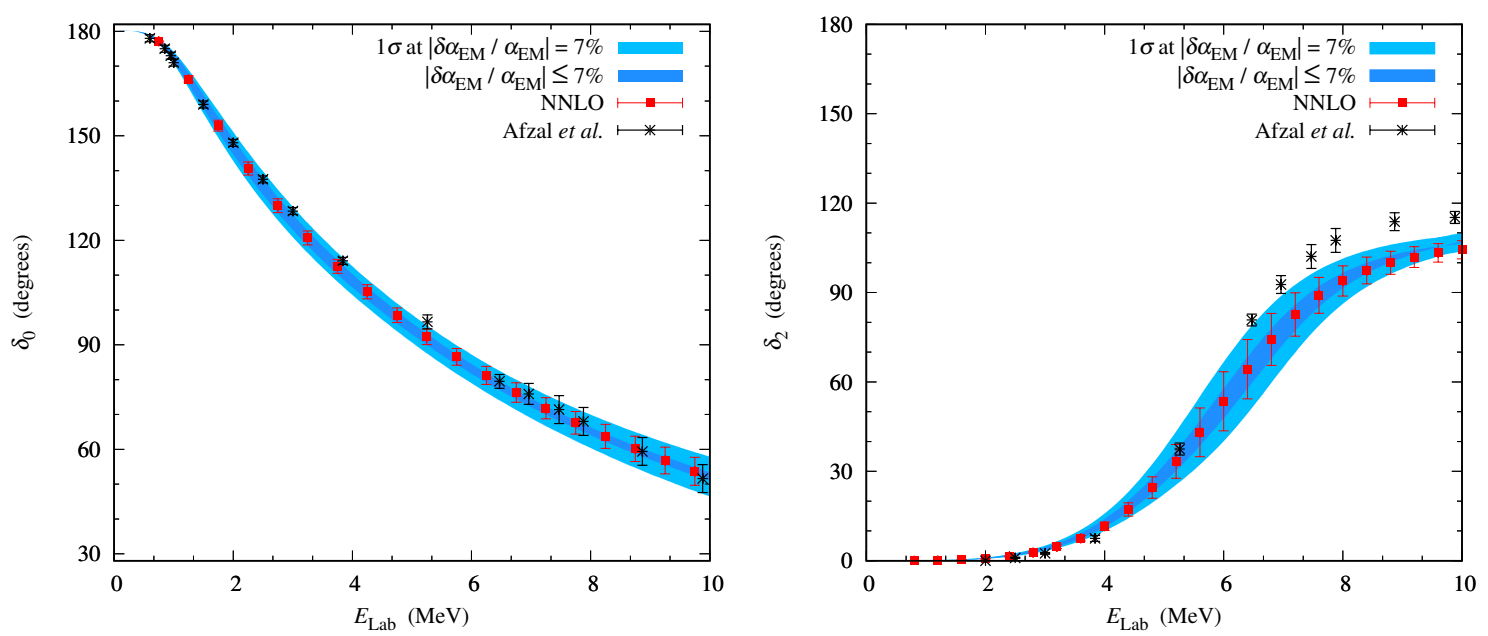

Figure 8. Dependence of the $\alpha$ - $\alpha$ phase shifts on the fine-structure constant at NNLO. Left panel: S-wave phase shift $\delta_{0}$ versus the energy in the laboratory system, $E_{\text {lab }}$. Right panel: D-wave phase shift $\delta_{0}$ versus the energy in the laboratory system, $E_{\text {lab }}$. The black crosses refer to the experimental data, the red squares are the results for $\delta \alpha_{\mathrm{EM}}=0$, the dark blue band corresponds to variations in $\alpha_{\mathrm{EM}} / \alpha_{\mathrm{EM}} \leq 7 \%(5 \%)$, for the S-(D-)wave and the light blue band represents the corresponding $1 \sigma$ error.

section 4 , we find that the phase shifts are little affected by variations in $\alpha_{\mathrm{EM}}$, as shown in figure 8 for the NNLO results. Here, variations of $\alpha_{\mathrm{EM}}$ up to $\pm 7 \%$ are displayed, where the upper (lower) part of the band refers to positive (negative) shifts in the fine-structure constant. We see that the variation in $\alpha_{\mathrm{EM}}$ has essentially no effect on the phase shifts. This can be explained as follows: by far the largest EM effect is the long-range Coulomb interaction between the two clusters. Now we are measuring the phase shifts with respect to the Coulomb-modified effective range expansion (see appendix $\mathrm{C}$ ), and thus this dominant effect is already taken care of. In contrast to the bound state energies (see section 8.2.1), the effect of the variation of the remaining, shorter-ranged EM corrections appears to be insignificant.

\subsubsection{Remarks on the $\theta$-dependence of alpha-alpha scattering}

In section 5 , we had shown that up to NLO, we can get the $\theta$-dependence of the $\alpha$ - $\alpha$ scattering phase shifts directly from the $\theta$-dependence of the pion mass. Therefore, we can directly translate the pion mass dependence of $\delta_{0,2}$ into a $\theta$-dependence. The depicted bands of the S-wave phase shifts for $\delta M_{\pi} / M_{\pi}=-3 \%$ and $-5 \%$ in figure 4 correspond to a variation of $\theta=0.7$ and 0.9 , respectively. At such values of $\theta$, the di-proton and the di-neutron are bound and element generation would proceed differently, for details see ref. [22].

We also note that a simultaneous variation of the light quark masses and $\theta$ can lead to a mutual (partial) compensation of effects, or to a mutual amplification. The latter case appears when $0<|\theta|<\pi$ and at the same time $\delta \hat{m} / \hat{m}<0$ as both result in a decrease of the pion mass. If one the other hand has $0<\delta \hat{m} / \hat{m} \leq 10 \%$ one can always find a value for $\theta$ such that $M_{\pi}(\hat{m}, \theta)=M_{\pi \text {,phys }}$ and nuclear physics would not be altered drastically (at least up to the order we are considering here). 


\section{Summary and outlook}

In this work, we have considered the fundamental process of $\alpha-\alpha$ scattering based on $a b$ initio calculations in the framework of Nuclear Lattice Effective Field Theory, both for the physical values of the light quark mass, the fine-structure constant, and the QCD $\theta$-angle, as well as for variations in these parameters. The main findings of this work can be summarized as follows:

- Due to improvements in the Adiabatic Projection Method compared to the pioneering study of $\alpha$ - $\alpha$ scattering in ref. [15], we obtain a very good description of the S- and D-wave phase shifts up to energies $E_{\text {lab }} \simeq 10 \mathrm{MeV}$ at NNLO in the chiral expansion.

- For the study of the variations under changes of the pion mass with $\left|\delta M_{\pi} / M_{\pi}\right| \leq 10 \%$, we rely on the pion mass dependent nuclear Hamiltonian worked out in ref. [11]. To this order, the ${ }^{8} \mathrm{Be}$ nucleus is slightly bound. In the S-wave phase shift, we find a dramatic effect (unbinding of the two-alpha system) for changes of $-5 \%$ and $-7 \%$ at NLO and NNLO, respectively. We have also considered the pion mass variation of the $\mathrm{S}$-wave effective range function, which is less sensitive to the binding issue and shows an added repulsion for negative pion mass shifts. This additional repulsion will certainly impact the position and the lifetime of ${ }^{8} \mathrm{Be}$. The pion mass variation on the D-wave is somewhat more pronounced, as seen by the effect on the corresponding resonance parameters and also by the D-wave effective range function.

- The dominant electromagnetic effect on the $\alpha$ - $\alpha$ scattering phase shifts is the longranged Coulomb potential that is included exactly by using a spherical wall with Coulomb boundary conditions. Taking this effect into account via the Coulombmodified ERE, we find very small effects of variations of $\alpha_{\mathrm{EM}}$ on the S- and D-wave phase shifts.

- We have shown that up-to-and-including NLO in the chiral expansion, the dependence of the $\alpha-\alpha$ scattering phase shifts on the QCD $\theta$-angle is entirely given by the $\theta$ dependence of the pion mass.

In summary, we find that $\alpha$ - $\alpha$ scattering (not unexpectedly) sets weaker constraints on the variation of the light quark masses and the fine-structure constant than that given by the closeness of the $3 \alpha$ threshold to the Hoyle state. However, as discussed in detail e.g. in refs. $[8,11]$, this requires stellar modelling which introduces some model-dependence. In contrast to that, the investigation of $\alpha-\alpha$ scattering discussed here is truly ab initio and not affected by such effects. Still, to further improve these calculations, a better determination of the pion mass dependence of the singlet and triplet NN scattering lengths from lattice QCD is mandatory. 


\section{Acknowledgments}

We are grateful for discussions with members of the Nuclear Lattice Effective Field Theory Collaboration. We gratefully acknowledge funding by the Deutsche Forschungsgemeinschaft (DFG, German Research Foundation) and the NSFC through the funds provided to the Sino-German Collaborative Research Center TRR110 "Symmetries and the Emergence of Structure in QCD" (DFG Project ID 196253076 — TRR 110, NSFC Grant No. 12070131001), the Chinese Academy of Sciences (CAS) President's International Fellowship Initiative (PIFI) (Grant No. 2018DM0034), Volkswagen Stiftung (Grant No. 93562), the European Research Council (ERC) under the European Union's Horizon 2020 research and innovation programme (grant agreement No. 101018170), the U.S. Department of Energy (DE-SC0013365 and DE-SC0021152) and the Nuclear Computational Low-Energy Initiative (NUCLEI) SciDAC-4 project (DE-SC0018083) and the Scientific and Technological Research Council of Turkey (TUBITAK project no. 120F341). The authors gratefully acknowledge the Gauss Centre for Supercomputing e.V. (www.gauss-centre.eu) for funding this project by providing computing time on the GCS Supercomputer JUWELS at Jülich Supercomputing Centre (JSC). Further computational resources were provided by the Oak Ridge Leadership Computing Facility through the INCITE award "Ab-initio nuclear structure and nuclear reactions", and by the JSC on the JURECA-DC supercomputer.

\section{A Details on the NN scattering parameters at varying pion mass}

Here, we give some details on how we extract the values of $\bar{A}_{s, t}$ from lattice data combined with EFT methods, closely following ref. [11]. In the earlier work [10] a combination of EFT and some modelling based on resonance saturation was used to pin down the pion mass dependence of the singlet and triplet inverse scattering lengths, which unavoidably induced some uncertainty that is difficult to control. However, ref. [51] proposed to use low-energy theorems to reconstruct the energy dependence of the NN scattering amplitude in a large kinematical domain from a single observable (such as the binding energy, scattering length, effective range) at a given fixed value of the pion mass [51]. Based on this, the lattice QCD data for the deuteron, the dineutron binding energy and the ERE parameters from refs. [52-56] are used to extract the quantities $\bar{A}_{s}$ and $\bar{A}_{t}$, as these appear to be mutually consistent. To perform interpolation between these five lattice-QCD points and the experimental values of the inverse scattering lengths, a simple quadratic ansatz is used:

$$
a_{s, t}^{-1}\left(M_{\pi}\right)=\left(a_{s, t}^{\mathrm{ph}}\right)^{-1}+a\left(M_{\pi}-M_{\pi}^{\mathrm{ph}}\right)+b\left(M_{\pi}-M_{\pi}^{\mathrm{ph}}\right)^{2} .
$$

The coefficients $a, b$ are then determined from a least square fit to the available values of $a_{s, t}^{-1}$ at heavier-than-physical pion masses. This leads to the values $\bar{A}_{s}=0.54$ and $\bar{A}_{t}=0.33$. A cubic extrapolation yields $\bar{A}_{s}=0.78$ and $\bar{A}_{t}=0.49$, and we take the difference as an estimation of the uncertainty in $\bar{A}_{s, t}$. In that way, we arrive at the values given in eq. (3.18). 

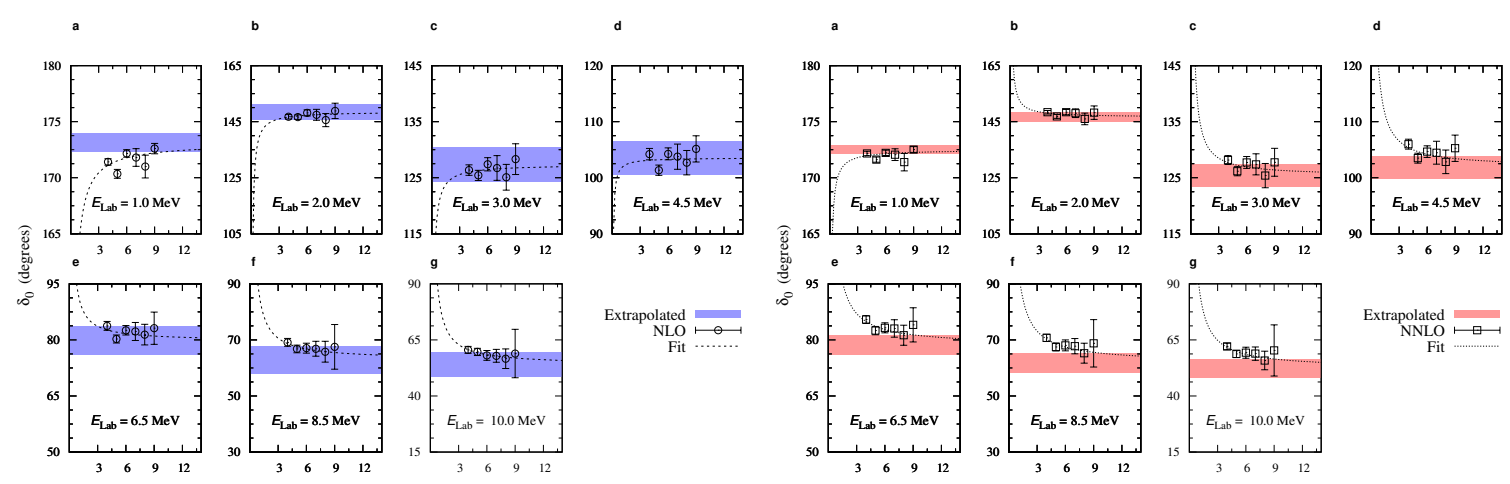

Figure 9. NLO (left panel) and NNLO (right panel) results for the S-wave phase shift $\delta_{0}$ versus $L_{t}$ for the lab energies $E_{\text {lab }}=1.0 \mathrm{MeV}, 2.0 \mathrm{MeV}, 3.0 \mathrm{MeV}, 4.5 \mathrm{MeV}, 6.5 \mathrm{MeV}, 8.5 \mathrm{MeV}$ and $10.0 \mathrm{MeV}$, respectively. The theoretical errors indicate the $1 \sigma$ uncertainty due to the MC errors. The dotted lines are fits to the data and used to extrapolate to the $L_{t} \rightarrow \infty$ limit. The hatched areas represent the $1 \sigma$ error of the extrapolation.
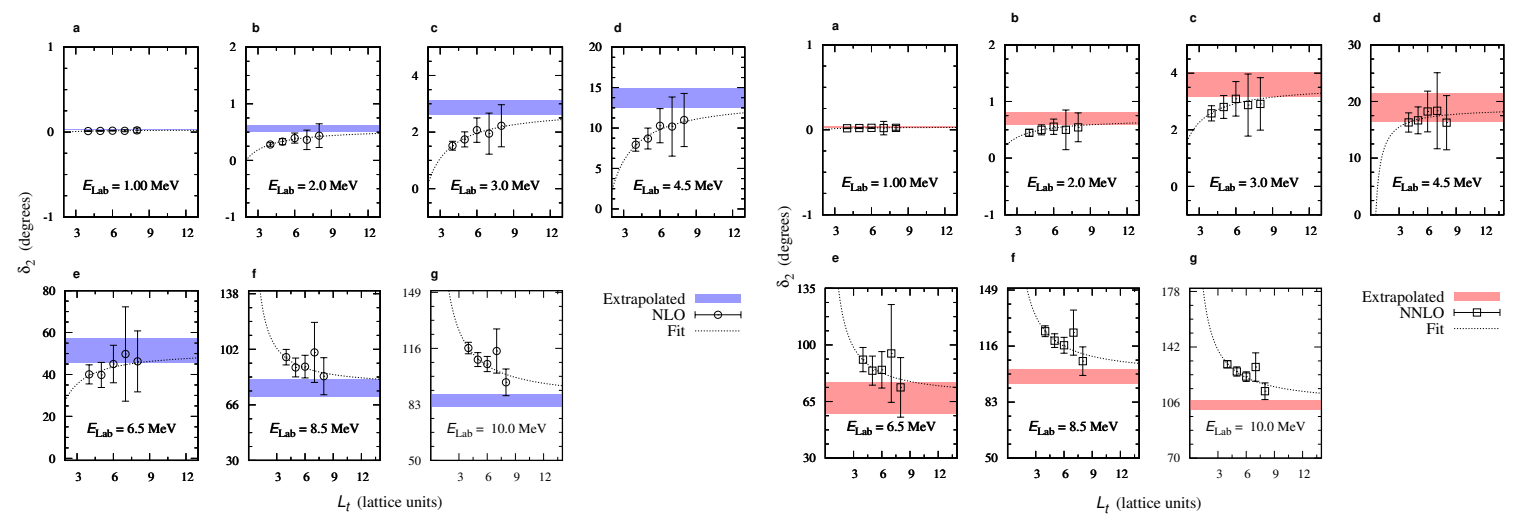

Figure 10. NLO (left panel) and NNLO (right panel) results for the D-wave phase shift $\delta_{2}$ versus $L_{t}$ for the lab energies $E_{\text {lab }}=1.0 \mathrm{MeV}, 2.0 \mathrm{MeV}, 3.0 \mathrm{MeV}, 4.5 \mathrm{MeV}, 6.5 \mathrm{MeV}, 8.5 \mathrm{MeV}$ and $10.0 \mathrm{MeV}$, respectively. The theoretical errors indicate the $1 \sigma$ uncertainty due to the MC errors. The dotted lines are fits to the data and used to extrapolate to the $L_{t} \rightarrow \infty$ limit. The hatched areas represent the $1 \sigma$ error of the extrapolation.

\section{B Euclidean time extrapolation}

We perform the AFQMC simulations and construct the radial adiabatic transfer matrices for the S-wave and D-wave channels from $L_{t}=4$ to $L_{t}=10$. Based on that, we compute the pertinent phase shifts with errors calculated using a jackknife analysis of the MC data. In figures 9 and 10 we show the NLO and NNLO results for the S- and D-wave phase shifts, respectively.

The dashed lines in these figures are the exponential curves used in the extrapolation to the limit $L_{t} \rightarrow \infty$. This is achieved by including some residual dependence from an excited state at an energy $\Delta E$ above the ground state, utilizing the ansatz:

$$
\delta_{\ell}\left(L_{t}, E\right)=\delta_{\ell}(E)+c_{\ell}(E) \exp \left[-\Delta E_{\ell} L_{t} a_{t}\right], \quad \ell=0,2,
$$


where the $c_{\ell}(E)$ and $\Delta E_{\ell}$ are fit parameters. As the gap between the $\alpha-\alpha$ threshold and these excited states is rather large, one finds a fast convergence as exhibited in these figures. There, the hatched areas represent the $1 \sigma$ deviation errors of the extrapolations, including the propagated MC errors of the data points.

\section{The Coulomb modified ERE}

Here, we collect the formulas for the Coulomb-modified ERE that was used above at NLO and NNLO. The Coulomb modified ERE takes the form [57-60]

$$
K_{\ell}(p)=C_{\eta, \ell}^{2} p^{2 \ell+1} \cot \left[\delta_{\ell}(p)\right]+\gamma h_{\ell}(p)=-\frac{1}{a_{\ell}}+\frac{1}{2} r_{\ell} p^{2}-\frac{1}{4} P_{\ell} p^{4}+\mathcal{O}\left(p^{6}\right),
$$

for a partial wave with angular momentum $\ell$ and $p$ is the relative momentum of the two scattering clusters. $K_{\ell}(p)$ is also called the effective-range function for angular momentum $\ell$. The factor $C_{\eta, \ell}^{2}$ is defined as

$$
C_{\eta, \ell}^{2}=\frac{2^{2 \ell}}{[(2 \ell+1) !]^{2}} C_{\eta, 0}^{2} \prod_{s=1}^{\ell}\left(s^{2}+\eta^{2}\right),
$$

where $C_{\eta, 0}^{2}$ is the conventional Sommerfeld factor,

$$
C_{\eta, 0}^{2}=\frac{2 \pi \eta}{e^{2 \pi \eta}-1},
$$

with $\eta=\gamma /(2 p)$. Here, $\gamma$ is the Coulomb parameter given by

$$
\gamma=2 \mu \alpha_{\mathrm{EM}} Z_{1} Z_{2},
$$

where $\mu$ is the reduced mass of the two-alpha system and $Z_{1}=Z_{2}=2$ are the charges of the two $\alpha$-particles. Finally, the factor $h_{\ell}(p)$ in (C.1) is given by

$$
h_{\ell}(p)=p^{2 \ell} \frac{C_{\eta, \ell}^{2}}{C_{\eta, 0}^{2}}(\operatorname{Re}[\psi(i \eta)]-\log |\eta|),
$$

where $\psi(z)=\Gamma^{\prime}(z) / \Gamma(z)$, in which the prime denotes differentiation.

\section{Bound state energies for varying pion masses}

Here, we collect the derivatives of the various ground state energies and the energy of the Hoyle state with respect to the pion mass as a function of the parameters $\bar{A}_{s}$ and $\bar{A}_{t}$, using the updated values for $x_{1}$ and $x_{2}$ collected in section 3 (for details, see ref. [10]),

$$
\begin{aligned}
& \left.\frac{\partial E_{4}}{\partial M_{\pi}}\right|_{M_{\pi}^{\mathrm{ph}}}=-0.339(5) \bar{A}_{s}-0.698(4) \bar{A}_{t}+0.042(10), \\
& \left.\frac{\partial E_{8}}{\partial M_{\pi}}\right|_{M_{\pi}^{\mathrm{ph}}}=-0.796(31) \bar{A}_{s}-1.584(22) \bar{A}_{t}+0.098(25), \\
& \left.\frac{\partial E_{12}}{\partial M_{\pi}}\right|_{M_{\pi}^{\mathrm{ph}}}=-1.519(27) \bar{A}_{s}-2.884(19) \bar{A}_{t}+0.174(46), \\
& \left.\frac{\partial E_{12}^{\star}}{\partial M_{\pi}}\right|_{M_{\pi}^{\mathrm{ph}}}=-1.589(12) \bar{A}_{s}-3.025(9) \bar{A}_{t}+0.194(47),
\end{aligned}
$$

where the error in the parenthesis is the combined statistical one from the AFQMC calculation and the systematic one due the uncertainties in $x_{1}$ and $x_{2}$. 
Open Access. This article is distributed under the terms of the Creative Commons Attribution License (CC-BY 4.0), which permits any use, distribution and reproduction in any medium, provided the original author(s) and source are credited.

\section{References}

[1] F. Hoyle, On Nuclear Reactions Occuring in Very Hot Stars. 1. The Synthesis of Elements from Carbon to Nickel, Astrophys. J. Suppl. 1 (1954) 121 [INSPIRE].

[2] S.A. Afzal, A.A.Z. Ahmad and S. Ali, Systematic Survey of the $\alpha$ - $\alpha$ Interaction, Rev. Mod. Phys. 41 (1969) 247 [INSPIRE].

[3] C.J. Hogan, Why the universe is just so, Rev. Mod. Phys. 72 (2000) 1149 [astro-ph/9909295] [INSPIRE].

[4] A.N. Schellekens, Life at the Interface of Particle Physics and String Theory, Rev. Mod. Phys. 85 (2013) 1491 [arXiv:1306.5083] [INSPIRE].

[5] U.-G. Meißner, Anthropic considerations in nuclear physics, Sci. Bull. 60 (2015) 43 [arXiv: 1409.2959] [INSPIRE].

[6] J.F. Donoghue, The Multiverse and Particle Physics, Ann. Rev. Nucl. Part. Sci. 66 (2016) 1 [arXiv: 1601.05136] [INSPIRE].

[7] F.C. Adams, The degree of fine-tuning in our universe — and others, Phys. Rept. 807 (2019) 1 [arXiv: 1902.03928] [INSPIRE].

[8] H. Oberhummer, A. Csoto and H. Schlattl, Stellar production rates of carbon and its abundance in the universe, Science $\mathbf{2 8 9}$ (2000) 88 [astro-ph/0007178] [INSPIRE].

[9] E. Epelbaum, H. Krebs, T.A. Lähde, D. Lee and U.-G. Meißner, Viability of Carbon-Based Life as a Function of the Light Quark Mass, Phys. Rev. Lett. 110 (2013) 112502 [arXiv: 1212.4181] [INSPIRE].

[10] E. Epelbaum, H. Krebs, T.A. Lähde, D. Lee and U.-G. Meißner, Dependence of the triple-alpha process on the fundamental constants of nature, Eur. Phys. J. A 49 (2013) 82 [arXiv: 1303.4856] [INSPIRE].

[11] T.A. Lähde, U.-G. Meißner and E. Epelbaum, An update on fine-tunings in the triple-alpha process, Eur. Phys. J. A 56 (2020) 89 [arXiv: 1906.00607] [InSPIRE].

[12] M. Pine, D. Lee and G. Rupak, Adiabatic projection method for scattering and reactions on the lattice, Eur. Phys. J. A 49 (2013) 151 [arXiv:1309.2616] [inSPIRE].

[13] S. Elhatisari and D. Lee, Fermion-dimer scattering using an impurity lattice Monte Carlo approach and the adiabatic projection method, Phys. Rev. C 90 (2014) 064001 [arXiv: 1407.2784] [INSPIRE].

[14] A. Rokash, M. Pine, S. Elhatisari, D. Lee, E. Epelbaum and H. Krebs, Scattering cluster wave functions on the lattice using the adiabatic projection method, Phys. Rev. C 92 (2015) 054612 [arXiv: 1505. 02967] [INSPIRE].

[15] S. Elhatisari et al., Ab initio alpha-alpha scattering, Nature 528 (2015) 111 [arXiv: 1506.03513] [INSPIRE].

[16] S. Elhatisari, D. Lee, U.-G. Meißner and G. Rupak, Nucleon-deuteron scattering using the adiabatic projection method, Eur. Phys. J. A 52 (2016) 174 [arXiv:1603.02333] [INSPIRE]. 
[17] K. Kravvaris, S. Quaglioni, G. Hupin and P. Navratil, Ab initio framework for nuclear scattering and reactions induced by light projectiles, arXiv: 2012.00228 [INSPIRE].

[18] P.F. Bedaque, T. Luu and L. Platter, Quark mass variation constraints from Big Bang nucleosynthesis, Phys. Rev. C 83 (2011) 045803 [arXiv:1012.3840] [InSPIRE].

[19] J.C. Berengut et al., Varying the light quark mass: impact on the nuclear force and Big Bang nucleosynthesis, Phys. Rev. D 87 (2013) 085018 [arXiv:1301.1738] [INSPIRE].

[20] J. Dragos, T. Luu, A. Shindler, J. de Vries and A. Yousif, Confirming the Existence of the strong CP Problem in Lattice QCD with the Gradient Flow, Phys. Rev. C 103 (2021) 015202 [arXiv: 1902.03254] [INSPIRE].

[21] L. Ubaldi, Effects of theta on the deuteron binding energy and the triple-alpha process, Phys. Rev. D 81 (2010) 025011 [arXiv:0811.1599] [INSPIRE].

[22] D. Lee, U.-G. Meißner, K.A. Olive, M. Shifman and T. Vonk, $\theta$-dependence of light nuclei and nucleosynthesis, Phys. Rev. Res. 2 (2020) 033392 [arXiv: 2006.12321] [INSPIRE].

[23] S. Elhatisari et al., Nuclear binding near a quantum phase transition, Phys. Rev. Lett. 117 (2016) 132501 [arXiv: 1602.04539] [INSPIRE].

[24] Y. Kanada-En'yo and D. Lee, Effective interactions between nuclear clusters, Phys. Rev. C 103 (2021) 024318 [arXiv: 2008.01867] [INSPIRE].

[25] A. Rokash, E. Epelbaum, H. Krebs and D. Lee, Effective forces between quantum bound states, Phys. Rev. Lett. 118 (2017) 232502 [arXiv:1612.08004] [INSPIRE].

[26] D.B. Kaplan and M.J. Savage, The Spin flavor dependence of nuclear forces from large $N$ QCD, Phys. Lett. B 365 (1996) 244 [hep-ph/9509371] [INSPIRE].

[27] D.B. Kaplan and A.V. Manohar, The Nucleon-nucleon potential in the $1 / N_{c}$ expansion, Phys. Rev. C 56 (1997) 76 [nucl-th/9612021] [InSPIRE].

[28] D. Lee et al., Hidden Spin-Isospin Exchange Symmetry, Phys. Rev. Lett. 127 (2021) 062501 [arXiv: 2010.09420] [INSPIRE].

[29] D. Lee, Lattice simulations for few- and many-body systems, Prog. Part. Nucl. Phys. 63 (2009) 117 [arXiv: 0804.3501] [INSPIRE].

[30] T.A. Lähde and U.-G. Meißner, Nuclear Lattice Effective Field Theory: An introduction, Lect. Notes Phys. 957 (2019) 1 [inSPIRE].

[31] M. Hoferichter, J. Ruiz de Elvira, B. Kubis and U.-G. Meißner, Roy-Steiner-equation analysis of pion-nucleon scattering, Phys. Rept. 625 (2016) 1 [arXiv:1510.06039] [INSPIRE].

[32] M. Hoferichter, J. Ruiz de Elvira, B. Kubis and U.-G. Meißner, Matching pion-nucleon Roy-Steiner equations to chiral perturbation theory, Phys. Rev. Lett. 115 (2015) 192301 [arXiv: 1507.07552] [INSPIRE].

[33] J. Gasser and H. Leutwyler, Chiral Perturbation Theory to One Loop, Annals Phys. 158 (1984) 142 [INSPIRE].

[34] J. Ruiz de Elvira, M. Hoferichter, B. Kubis and U.-G. Meißner, Extracting the $\sigma$-term from low-energy pion-nucleon scattering, J. Phys. G 45 (2018) 024001 [arXiv:1706.01465] [INSPIRE].

[35] C.C. Chang et al., A per-cent-level determination of the nucleon axial coupling from quantum chromodynamics, Nature 558 (2018) 91 [arXiv:1805.12130] [INSPIRE]. 
[36] E. Epelbaum and U.-G. Meißner, Isospin-violating nucleon-nucleon forces using the method of unitary transformation, Phys. Rev. C 72 (2005) 044001 [nucl-th/0502052] [INSPIRE].

[37] R. Brower, S. Chandrasekharan, J.W. Negele and U.J. Wiese, QCD at fixed topology, Phys. Lett. B 560 (2003) 64 [hep-lat/0302005] [INSPIRE].

[38] A.V. Smilga, QCD at theta similar to pi, Phys. Rev. D 59 (1999) 114021 [hep-ph/9805214] [INSPIRE].

[39] T. Vonk, F.-K. Guo and U.-G. Meißner, Aspects of the QCD A-vacuum, JHEP 06 (2019) 106 [Erratum ibid. 10 (2019) 028] [arXiv:1905.06141] [INSPIRE].

[40] V. Bernard, N. Kaiser and U.-G. Meißner, Aspects of chiral pion-nucleon physics, Nucl. Phys. A 615 (1997) 483 [hep-ph/9611253] [INSPIRE].

[41] T. Vonk, Studies on the QCD $\theta$-vacuum in chiral perturbation theory, MSc Thesis, University of Bonn (2019).

[42] S. Elhatisari, Adiabatic projection method with Euclidean time subspace projection, Eur. Phys. J. A 55 (2019) 144 [arXiv: 1906.01046] [InSPIRE].

[43] B.-N. Lu, T.A. Lähde, D. Lee and U.-G. Meißner, Precise determination of lattice phase shifts and mixing angles, Phys. Lett. B 760 (2016) 309 [arXiv:1506.05652] [INSPIRE].

[44] J. Carlson, V.R. Pandharipande and R.B. Wiringa, Variational calculations of resonant states in ${ }^{4} \mathrm{He}$, Nucl. Phys. A 424 (1984) 47 [InSPIRE].

[45] B. Borasoy, E. Epelbaum, H. Krebs, D. Lee and U.-G. Meißner, Two-particle scattering on the lattice: Phase shifts, spin-orbit coupling, and mixing angles, Eur. Phys. J. A 34 (2007) 185 [arXiv:0708.1780] [INSPIRE].

[46] R. Higa, H.W. Hammer and U. van Kolck, $\alpha \alpha$ Scattering in Halo Effective Field Theory, Nucl. Phys. A 809 (2008) 171 [arXiv:0802.3426] [INSPIRE].

[47] G. Rasche, Effective range analysis of s-and d-wave $\alpha$ - $\alpha$ scattering, Nucl. Phys. A 94 (1967) 301 [Erratum ibid. 119 (1968) 692] [INSPIRE].

[48] G. Hupin, S. Quaglioni and P. Navrátil, Predictive theory for elastic scattering and recoil of protons from ${ }^{4} H e$, Phys. Rev. C 90 (2014) 061601 [arXiv: 1409.0892] [InSPIRE].

[49] M. Livio, D. Hollowell, A. Weiss and J.W. Truran, The anthropic significance of the existence of an excited state of ${ }^{12} C$, Nature 340 (1989) 281.

[50] S. Weinberg, Facing Up, Harvard University Press, Cambridge, Massachusetts (2001).

[51] V. Baru, E. Epelbaum, A.A. Filin and J. Gegelia, Low-energy theorems for nucleon-nucleon scattering at unphysical pion masses, Phys. Rev. C 92 (2015) 014001 [arXiv:1504.07852] [INSPIRE].

[52] NPLQCD collaboration, The Deuteron and Exotic Two-Body Bound States from Lattice QCD, Phys. Rev. D 85 (2012) 054511 [arXiv:1109.2889] [INSPIRE].

[53] T. Yamazaki, K.-i. Ishikawa, Y. Kuramashi and A. Ukawa, Study of quark mass dependence of binding energy for light nuclei in $2+1$ flavor lattice QCD, Phys. Rev. D 92 (2015) 014501 [arXiv: 1502.04182] [INSPIRE].

[54] K. Orginos, A. Parreno, M.J. Savage, S.R. Beane, E. Chang and W. Detmold, Two nucleon systems at $m_{\pi} \sim 450 \mathrm{MeV}$ from lattice QCD, Phys. Rev. D 92 (2015) 114512 [Erratum ibid. 102 (2020) 039903] [arXiv: 1508.07583] [INSPIRE]. 
[55] NPLQCD collaboration, Nucleon-Nucleon Scattering Parameters in the Limit of SU(3) Flavor Symmetry, Phys. Rev. C 88 (2013) 024003 [arXiv: 1301.5790] [inSPIRE].

[56] T. Yamazaki, K.-i. Ishikawa, Y. Kuramashi and A. Ukawa, Helium nuclei, deuteron and dineutron in $2+1$ flavor lattice QCD, Phys. Rev. D 86 (2012) 074514 [arXiv:1207.4277] [INSPIRE].

[57] H.A. Bethe, Theory of the Effective Range in Nuclear Scattering, Phys. Rev. 76 (1949) 38 [INSPIRE].

[58] J.D. Jackson and J.M. Blatt, The Interpretation of Low Energy Proton-Proton Scattering, Rev. Mod. Phys. 22 (1950) 77 [INSPIRE].

[59] H. van Haeringen and L.P. Kok, Modified effective range function, Phys. Rev. A 26 (1982) 1218 [INSPIRE].

[60] S. König, D. Lee and H.W. Hammer, Causality constraints for charged particles, J. Phys. G 40 (2013) 045106 [arXiv:1210.8304] [INSPIRE]. 